\title{
Confinement effects on electron and phonon degrees of freedom in nanofilm superconductors: A Green function approach
}

\author{
R. Saniz, B. Partoens, and F. M. Peeters \\ Departement Fysica, Universiteit Antwerpen, Groenenborgerlaan 171, B-2020 Antwerpen, Belgium
}

(Received 1 June 2012; revised manuscript received 18 January 2013; published 25 February 2013)

\begin{abstract}
The Green function approach to the Bardeen-Cooper-Schrieffer theory of superconductivity is used to study nanofilms. We go beyond previous models and include effects of confinement on the strength of the electronphonon coupling as well as on the electronic spectrum and on the phonon modes. Within our approach, we find that in ultrathin films, confinement effects on the electronic screening become very important. Indeed, contrary to what has been advanced in recent years, the sudden increases of the density of states when new bands start to be occupied as the film thickness increases, tend to suppress the critical temperature rather than to enhance it. On the other hand, the increase of the number of phonon modes with increasing number of monolayers in the film leads to an increase in the critical temperature. As a consequence, the superconducting critical parameters in such nanofilms are determined by these two competing effects. Furthermore, in sufficiently thin films, the condensate consists of well-defined subcondensates associated with the occupied bands, each with a distinct coherence length. The subcondensates can interfere constructively or destructively giving rise to an interference pattern in the Cooper pair probability density.
\end{abstract}

DOI: 10.1103/PhysRevB.87.064510

PACS number(s): 74.20.-z, 74.78.-w, 74.81.-g

\section{INTRODUCTION}

In 1963, Blatt and Thompson studied theoretically very thin, nanoscale, films within the Bardeen-Cooper-Schrieffer (BCS) theory of superconductivity. ${ }^{1,2}$ To study such an inhomogeneous system, they essentially applied a method first advanced by Anderson in his study of dirty superconductors. ${ }^{3,4}$ An interesting finding in their work was that, as a function of film thickness, the critical temperature $\left(T_{c}\right)$ and gap value exhibit very sharp oscillations, which are stronger the thinner the nanofilm and which the authors referred to as shape resonances. Briefly, in a nanofilm, the electron motion in the direction transverse to the film is strongly quantized, resulting in quantum well states, which, nevertheless, move as free particles in the directions parallel to the film. This results in an energy spectrum consisting of a discrete set of two-dimensional bands. Band occupancy depends on the nanofilm thickness. With increasing nanofilm thickness, the number of occupied bands increases by one in a repeated fashion. This results in repeated and sudden jumps of the electronic density of states at the Fermi level with increasing film thickness and leads to the sharp oscillations found by Blatt and Thompson. Interestingly, their model resulted in $T_{c}$ 's and gap values that could be more than twice the bulk values. Later, similar studies explored further the consequences of the size quantization of the electronic degrees of freedom. ${ }^{5,6}$ However, quantum size effects can manifest themselves more broadly, affecting, e.g., the phononic degrees of freedom as well. This has been considered in Ref. 7, where the quantization of the phonon spectra in the nanofilm was taken into account. This leads to multigap superconductivity and to a modification of the gap and critical temperature dependencies on nanofilm thickness. As shown in this work, however, the picture is incomplete if no account is taken of the effect of confinement on the electron-phonon coupling strength.

On the experimental side, there were early reports of enhanced $T_{c}$ 's in $\mathrm{Al}$ thin films ${ }^{8}$ or of oscillations in the $T_{c}$ of $\mathrm{Sn}$ thin films as a function of their thickness. ${ }^{9}$ But consensus regarding the observation of quantum size effects in nanofilms has required the advent of high-quality thin film growth techniques. Oscillations in the $T_{c}$ and/or gap values as a function of the number of monolayers have been reported in recent years for $\mathrm{Pb}$ nanofilms deposited on a Si(111) substrate. ${ }^{10-12}$ Strikingly, superconductivity was found to persist even in films only one monolayer thick. ${ }^{13}$ Also, in $\mathrm{Al}$ nanofilms, the superconducting gap was found to increase with decreasing film thickness, reaching values nearly $75 \%$ higher than in the bulk for a thickness of $5 \mathrm{~nm} .{ }^{14}$ In the case of $\mathrm{Pb}$ nanofilms, on the other hand, the critical parameters reported have lower values than their bulk counterparts. ${ }^{10-12}$ In several of the theoretical works, ${ }^{6,15,16}$ efforts were made to explain the oscillation of the critical parameters in $\mathrm{Pb}$ nanofilms essentially in terms of the oscillations discussed by Blatt and Thompson. Indeed, these works focus again on the electronic degrees of freedom and are based either on the Anderson method mentioned above ${ }^{6}$ or on the Bogoliubov-de Gennes method. ${ }^{15,16}$ A more recent comparison between theory, based on the latter, and experiment can be found in Ref. 17. Studies based on density functional theory have also been carried out on $\mathrm{Al}$ nanofilms ${ }^{18}$ as well as on $\mathrm{Pb}$ nanofilms. ${ }^{19}$ One of the main findings of these latter works is that the surface phonon modes induced by confinement contribute to electron-phonon coupling in opposite ways in $\mathrm{Al}$ and in $\mathrm{Pb}$. In the latter, the coupling becomes weaker, while in the former, it becomes stronger, thereby explaining the enhancement of the $T_{c}$ in $\mathrm{Al}$ nanofilms and its reduction in $\mathrm{Pb}$ nanofilms.

The Green function formalism of the BCS theory of superconductivity was first advanced by Gor'kov for homogeneous systems. ${ }^{20}$ The present work is based on a reformulation, which is particularly convenient to study inhomogeneous systems. In a previous report (Ref. 21, hereafter referred to as I), we applied it to study superconductivity in nanowires. The reformulation is based on Anderson's method. ${ }^{3}$ It consists essentially in first solving the Hamiltonian for the normal state and then adding a phenomenological effective attractive (or pairing) interaction and solving the problem within a mean- 
field approximation. Several years ago, Tanaka and Marsiglio showed that the Anderson approach compares well with the Bogoliubov-de Gennes approach, in the weak coupling case, using an attractive Hubbard model to study inhomogeneity effects in superconductors. ${ }^{4}$ The same conclusion is reached in I, where the agreement between the two approaches using similar parameters was found to be excellent. Here, we show that this approach allows to shed new light on several aspects of superconductivity in nanofilms, and with a computational effort far less costly than the Bogoliubov-de Gennes or density functional approaches. The present work differs from the previous studies ${ }^{1,2,5-7,15,16}$ in that account is taken of the effects of confinement not only on the electronic spectrum and on the phonon modes but also on the strength of the electron-phonon coupling. We find that, in contrast to what is currently usually assumed, ${ }^{6,9-12,15,16}$ the confinement effects on superconductivity in very thin nanofilms do not always correlate with the electron density of states. A critical finding is that when the film thickness is below a couple of tens of monolayers, at normal metallic densities, confinement effects on the screened Coulomb interaction between the electrons and the ionic background become very important. Indeed, each time when the number of occupied bands increases with nanofilm thickness, the screened electron-electron interaction weakens because screening increases, causing electronphonon coupling to decrease. This would lead the critical parameters to drop rather than to increase. Remarkably, this is strongly offset when the number of monolayers increases as the number of active phonon modes contributing to the electron-phonon coupling increases. Thus the observed dependence of the critical parameters on the monolayer number is determined by a strong interplay between the two effects.

Another very interesting aspect to consider in nanofilms is the effect of confinement on the superconducting condensate. Because translational symmetry is broken, the Cooper pair wave function depends on both the relative and center of mass coordinates of the pair. Thus, for instance, although the coherence length is determined by the behavior of the pair wave function as a function of the relative coordinates, it is a local property, in the sense that it depends on the position of the center of mass. This is typical for superconductivity in inhomogeneous systems, such as nuclei ${ }^{22,23}$ and high- $T_{c}$ superconductors. ${ }^{24,25}$ We show here that in sufficiently thin films, the condensate is composed of well-defined subcondensates, each associated with an occupied band and its corresponding gap. These produce an interference pattern in the Cooper pair wave function probability density with regions depleted of pairs. Moreover, the different subcondensates generally exhibit different coherence lengths. This is quite similar to the case of two-gap bulk superconductors, such as $\mathrm{MgB}_{2}$, where the two gaps were shown to lead to different length scales. ${ }^{26,27}$

In Sec. II, below, we present the model system studied. Also, for completeness, the more general derivation of the gap equation presented in I is specialized to the case of nanofilms. Section III presents the derivation of the effective electron-electron interaction taking into account the effects of confinement both on the phonon spectrum and on the strength of the electron-phonon coupling. The resulting effects on the nanofilm thickness dependence of $T_{c}$ and gap values are analyzed in Sec. IV. In Sec. V, we discuss the consequences of confinement on the spatial behavior of the condensate. The present work is summarized in Sec. VI. In the appendixes, we present some of the technical steps in the derivation of the dielectric screening (see Appendix A) and more details regarding the calculation of the Cooper pair density and of the coherence lengths (see Appendix B).

\section{THE GAP EQUATION}

Consider the BCS grand-canonical Hamiltonian ${ }^{28}$

$$
\begin{aligned}
\hat{H}= & \sum_{\sigma} \int d \mathbf{r} \hat{\psi}_{\sigma}^{\dagger}(\mathbf{r})\left[H_{0}(\mathbf{r})-\mu\right] \hat{\psi}_{\sigma}(\mathbf{r}) \\
& +\frac{1}{2} \int d \mathbf{r} d \mathbf{r}^{\prime} \hat{\psi}_{\uparrow}^{\dagger}(\mathbf{r}) \hat{\psi}_{\downarrow}^{\dagger}\left(\mathbf{r}^{\prime}\right) v_{\mathrm{eff}}\left(\mathbf{r}, \mathbf{r}^{\prime}\right) \hat{\psi}_{\downarrow}\left(\mathbf{r}^{\prime}\right) \hat{\psi}_{\uparrow}(\mathbf{r}),
\end{aligned}
$$

where $H_{0}$ is the independent electron Hamiltonian, $\mu$ is the chemical potential (Fermi level), and $\hat{\psi}_{\sigma}^{\dagger}\left(\hat{\psi}_{\sigma}\right)$ is the creation (destruction) field operator for an electron with spin $\sigma \cdot v_{\text {eff }}$ is the effective attractive interaction, coupling electrons of opposite spin. Its precise form need not be specified for now. Section III is devoted to that subject. As previous authors, we consider a free-standing nanofilm. 2,5-7,15,18,19 Because the impurity scattering length in high-quality nanofilms is much larger than the film thickness, the nanofilms can be treated in the clean limit. ${ }^{16}$ The system of electrons in the nanofilm is described (in the normal state) by assuming a model Hamiltonian $H_{0}=-\hbar^{2} \nabla^{2} / 2 m+V$, where $V$ is a potential well defined by two large planes of side $L$, a distance $d$ apart, such that $V[\mathbf{r} \equiv(\rho, z)]=0$ for $0 \leqslant z \leqslant$ $d$, and $\infty$ otherwise., ${ }^{2,7}$ The (orthonormal) eigenstates of $H_{0}$ are given by $\langle\mathbf{r} \mid \mathbf{q} n\rangle=\psi_{\mathbf{q} n}(\mathbf{r})=\left(2 / L^{2} d\right)^{1 / 2} e^{i \mathbf{q} \cdot \boldsymbol{\rho}} \sin \frac{n \pi z}{d}$, where $\mathbf{q}=2 \pi(l, m) / L$ with $l, m \in \mathbb{Z}$, i.e., assuming periodic boundary conditions in the $x O y$ plane (the limit $L \rightarrow \infty$ is taken at the end of the calculations). The energy levels are given by a set of nonintersecting paraboloids, namely, $E_{\mathbf{q} n}=\hbar^{2}\left(q^{2}+n^{2} \pi^{2} / d^{2}\right) / 2 m$. Hence, given a Fermi level $\mu$, the Fermi surface is broken into a set of concentric circumferences of radii $q_{\mathrm{F}}^{(n)}=\left(2 m \mu / \hbar^{2}-n^{2} \pi^{2} / d^{2}\right)^{1 / 2}$. Following Anderson, ${ }^{3}$ we assume that the effective interaction $v_{\text {eff }}$ couples only time-reversed states. Here, these correspond to $|\mathbf{q} n\rangle$ and $|-\mathbf{q} n\rangle$. Note that this means, in particular, that both electrons in a pair necessarily belong to the same electronic band.

A Hamiltonian, such as the above, was solved quite generally in I. Here, the main points are emphasized, while introducing the notation used further on. One considers the equation of motion for the single-electron temperature Green function,

$$
\mathcal{G}\left(\mathbf{r} \mathbf{r}^{\prime}, \tau\right)=-\left\langle T_{\tau} \hat{\psi}_{\uparrow}(\mathbf{r} \tau) \hat{\psi}_{\uparrow}^{\dagger}\left(\mathbf{r}^{\prime} 0\right)\right\rangle,
$$

and seeks a solution within a mean-field approximation by introducing the Gor'kov functions:

$$
\begin{aligned}
& \mathcal{F}\left(\mathbf{r r}^{\prime}, \tau\right)=-\left\langle T_{\tau} \hat{\psi}_{\uparrow}(\mathbf{r} \tau) \hat{\psi}_{\downarrow}\left(\mathbf{r}^{\prime} 0\right)\right\rangle, \\
& \mathcal{F}^{\dagger}\left(\mathbf{r r}^{\prime}, \tau\right)=-\left\langle T_{\tau} \hat{\psi}_{\downarrow}^{\dagger}(\mathbf{r} \tau) \hat{\psi}_{\uparrow}^{\dagger}\left(\mathbf{r}^{\prime} 0\right)\right\rangle .
\end{aligned}
$$


It proves useful to expand the field operators in terms of the eigenstates of $H_{0}$. In the present case, $\hat{\psi}_{\sigma}(\mathbf{r} \tau)=$ $\sum_{\mathbf{q} n} \psi_{\mathbf{q} n}(\mathbf{r}) c_{\mathbf{q} n \sigma}(\tau)$ and $\hat{\psi}_{\sigma}^{\dagger}(\mathbf{r} \tau)=\sum_{\mathbf{q} n} \psi_{\mathbf{q} n}^{*}(\mathbf{r}) c_{\mathbf{q} n \sigma}^{\dagger}(\tau)$. Operator $c_{\mathbf{q} n \sigma}^{\dagger}\left(c_{\mathbf{q} n \sigma \sigma}\right)$ creates (destroys) an electron in state $|n \mathbf{q}\rangle$, with spin $\sigma$. Thus, for instance, one has the expansion

$$
\mathcal{F}^{\dagger}\left(\mathbf{r} \mathbf{r}^{\prime}, \tau\right)=\sum_{\mathbf{q} n \mathbf{q}^{\prime} n^{\prime}} \psi_{\mathbf{q} n}^{*}(\mathbf{r}) \psi_{\mathbf{q}^{\prime} n^{\prime}}^{*}\left(\mathbf{r}^{\prime}\right) \mathcal{F}^{\dagger}\left(\mathbf{q} n \mathbf{q}^{\prime} n^{\prime}, \tau\right),
$$

with $\mathcal{F}^{\dagger}\left(\mathbf{q} n \mathbf{q}^{\prime} n^{\prime}, \tau\right)=-\left\langle T_{\tau} c_{\mathbf{q} n \downarrow}^{\dagger}(\tau) c_{\mathbf{q}^{\prime} n^{\prime} \uparrow}^{\dagger}(0)\right\rangle$. Also, the solution is most easily found in terms of the coefficients of the Fourier representation in $\tau$ of the above functions, e.g., $\mathcal{F}^{\dagger}\left(\mathbf{q} n \mathbf{q}^{\prime} n^{\prime}, \tau\right)=\frac{1}{\beta \hbar} \sum_{p} e^{-t \omega_{p} \tau} \tilde{\mathcal{F}}^{\dagger}\left(\mathbf{q} n \mathbf{q}^{\prime} n^{\prime}, \omega_{p}\right)$, where $\beta=$ $1 / k_{B} T$. As shown in I, for an effective interaction coupling only time-reversed states, the solution can be written as

$$
\begin{gathered}
\tilde{\mathcal{G}}\left(\mathbf{q} n \mathbf{q}^{\prime} n^{\prime}, \omega_{p}\right)=-\delta_{\mathbf{q} n \mathbf{q}^{\prime} n^{\prime}} \frac{i \omega_{p}+\epsilon_{\mathbf{q} n} / \hbar}{\omega_{p}^{2}+\xi_{\mathbf{q} n}^{2} / \hbar^{2}}, \\
\tilde{\mathcal{F}}^{\dagger}\left(\mathbf{q} n \mathbf{q}^{\prime} n^{\prime}, \omega_{p}\right)=\delta_{-\mathbf{q} n} \mathbf{q}^{\prime} n^{\prime} \frac{\Delta^{*}(\mathbf{q} n) / \hbar}{\omega_{p}^{2}+\xi_{\mathbf{q} n}^{2} / \hbar^{2}},
\end{gathered}
$$

where $\xi_{\mathbf{q} n}^{2}=\epsilon_{\mathbf{q} n}^{2}+|\Delta(\mathbf{q} n)|^{2}$ and $\epsilon_{\mathbf{q} n}=E_{\mathbf{q} n}-\mu$. The parameters $\Delta(\mathbf{q} n)$ are defined by

$$
\Delta(\mathbf{q} n)=\sum_{\mathbf{q}^{\prime} n^{\prime}} V_{\mathbf{q} n \mathbf{q}^{\prime} n^{\prime}} \mathcal{F}\left(-\mathbf{q}^{\prime} n^{\prime} \mathbf{q}^{\prime} n^{\prime}, 0\right),
$$

with $V_{\mathbf{q} n \mathbf{q}^{\prime} n^{\prime}} \equiv-\left\langle\mathbf{q} n,-\mathbf{q} n\left|v_{\text {eff }}\right| \mathbf{q} n^{\prime},-\mathbf{q}^{\prime} n^{\prime}\right\rangle$ the matrix elements of the effective interaction (the sign of the interaction is introduced for notational convenience). One can see from the above that $\Delta(\mathbf{q} n)$ represents a momentum- and banddependent energy gap. Combining their definition with the above solution for $\tilde{\mathcal{F}}$, one obtains the gap equation

$$
\Delta(\mathbf{q} n)=\sum_{\mathbf{q}^{\prime} n^{\prime}} V_{\mathbf{q} n, \mathbf{q}^{\prime} n^{\prime}} \Delta\left(\mathbf{q}^{\prime} n^{\prime}\right) \frac{1}{2 \xi_{\mathbf{q}^{\prime} n^{\prime}}} \tanh \frac{\xi_{\mathbf{q}^{\prime} n^{\prime}}}{2 k_{\mathrm{B}} T} .
$$

$\Delta(\mathbf{q} n)$ depends implicitly on temperature. To solve the gap equation, one still has to specify the matrix elements, $V_{\mathbf{q} n, \mathbf{q}^{\prime} n^{\prime}}$, of the effective interaction. We deduce below the specific form of $v_{\text {eff }}$ used in this work.

\section{EFFECTIVE ELECTRON-ELECTRON INTERACTION IN A NANOFILM}

In the BCS theory for homogeneous systems, as presented in most textbooks (see, e.g., Refs. 28 and 29), the effective attractive interaction between electrons of opposite spin is given by an attractive contact potential, $v_{\text {eff }}\left(\mathbf{r}, \mathbf{r}^{\prime}\right)=-g \delta(\mathbf{r}-$ $\left.\mathbf{r}^{\prime}\right) .{ }^{30}$ The interaction strength is given by the square of the electron-phonon coupling constant $\gamma$, that is, $g=\gamma^{2}$. Important from the point of view of the present work is that $\gamma$ is proportional to the screened Coulomb interaction. ${ }^{28}$

Here, we derive the formal effects of confinement on electron-phonon coupling, as well as the consequences on the phonon-mediated pairing interaction. In the previous section, the effects of confinement on the electron system in the nanofilm were already considered. The first section below presents the confined phonon system and describes how it modifies the form of the electron-phonon coupling. In the second section, we consider the effect of confinement on the strength of the electron-phonon coupling, i.e., we study the screened Coulomb interaction in a nanofilm. Finally, the third section is devoted to the consequent modification of the effective electron-electron interaction.

\section{A. Electron-phonon interaction}

\section{Phonon spectrum}

We use the uniform, elastic background model to describe the phonon system, paralleling the formulation of Fetter and Walecka. $^{28}$ If $\mathbf{d}=\mathbf{d}(\mathbf{r}, t)$ is the displacement field, characterizing the displacement from the equilibrium position, we consider the wave equation

$$
\frac{1}{c^{2}} \frac{\partial^{2}}{\partial t^{2}} \mathbf{d}(\mathbf{r}, t)-\nabla^{2} \mathbf{d}(\mathbf{r}, t)=0 .
$$

Here, $c=\sqrt{B / M n_{0}}$ is the speed of sound with $B$ the bulk modulus and $M$ and $n_{0}$ the background atomic mass and particle density, respectively (i.e., $M n_{0}$ is the background mass density); below, we write $\varrho_{0}=M n_{0}$. To describe sound waves, we take the ansatz

$$
\mathbf{d}(\mathbf{r}, t)=e^{i \mathbf{q} \cdot \rho-i \omega t} \hat{\mathbf{q}} \sin \frac{n \pi z}{d},
$$

where, again, $\mathbf{q}$ is the momentum parallel to the plane of the film. It is easy to verify that the wave equation above is satisfied by $\mathbf{d}$ with $\omega^{2}=\omega_{n q}^{2} \equiv c^{2}\left(q^{2}+n^{2} \pi^{2} / d^{2}\right) .{ }^{31}$ We now define the quantized displacement field as

$$
\begin{aligned}
\hat{\mathbf{d}}(\mathbf{r}, t)= & -\frac{i}{c \sqrt{\varrho_{0}}} \sum_{n \mathbf{q}}\left(\frac{\hbar \omega_{n q}}{2 V}\right)^{1 / 2} \frac{\hat{\mathbf{q}}}{q} \\
& \times\left(b_{n \mathbf{q}} e^{i \mathbf{q} \cdot \boldsymbol{\rho}-i \omega_{n q} t}-b_{n \mathbf{q}}^{\dagger} e^{-i \mathbf{q} \cdot \boldsymbol{\rho}+i \omega_{n q} t}\right) \sin \frac{n \pi z}{d},
\end{aligned}
$$

where $V$ is the volume of the system and $k=\sqrt{q^{2}+n^{2} \pi^{2} / d^{2}}$ for short. The operators $b_{n \mathbf{q}}^{\dagger}$ and $b_{n \mathbf{q}}$ create and destroy, respectively, a phonon with momentum $\mathbf{q}$ and energy $\hbar \omega_{n q}$.

\section{Electron-phonon interaction}

The electron-phonon interaction Hamiltonian can be written as (cf. Ref. 28)

$$
\hat{H}_{\mathrm{el}-\mathrm{ph}}=\int d^{3} r d^{3} r^{\prime} \hat{\varrho}(\mathbf{r}) v^{\mathrm{scr}}\left(\mathbf{r}, \mathbf{r}^{\prime}\right) . \delta \hat{\varrho}_{b}\left(\mathbf{r}^{\prime}\right) .
$$

We consider the different factors in the integrand one at the time. The first factor, $\hat{\varrho}$, is the electron charge density operator. In terms of the electron field operators, it is written $\hat{\varrho}(\mathbf{r})=-e \sum_{\sigma} \hat{\psi}_{\sigma}^{\dagger}(\mathbf{r}) \hat{\psi}_{\sigma}(\mathbf{r})$, with $e$ the magnitude of the electron charge. The second factor, $v^{\mathrm{scr}}$, is the screened Coulomb potential. Because of translational invariance along the $x O y$ plane, without loss of generality, it can be expanded in terms of the single-electron states as $v^{\mathrm{scr}}\left(\mathbf{r}, \mathbf{r}^{\prime}\right)=$ $\sum_{n n^{\prime} \mathbf{q}} \psi_{n \mathbf{q}}(\mathbf{r}) \psi_{n^{\prime} \mathbf{q}}^{*}\left(\mathbf{r}^{\prime}\right) v_{n n^{\prime} \mathbf{q}}^{\text {scr }}$. Finally, the third factor in Eq. (12) is the background charge density fluctuation, $\delta \hat{\varrho}_{b}=z e \delta \hat{n}(\mathbf{r})$, with $z$ the valence of the background ions and $\delta \hat{n}$ the background particle density fluctuation operator. Assuming a Debye model for the elastic dynamics of the background, ${ }^{28}$ 
it is given by

$$
\begin{aligned}
\delta \hat{\varrho}_{b}(\mathbf{r})= & -z e n_{0} \nabla \cdot \hat{\mathbf{d}}(\mathbf{r}) \\
= & -\frac{z e n_{0}}{c \sqrt{\varrho_{0}}} \sum_{n \mathbf{q}}\left(\frac{\hbar \omega_{n q}}{2 V}\right)^{1 / 2}\left(b_{n \mathbf{q}} e^{i \mathbf{q} \cdot \boldsymbol{\rho}}+b_{n \mathbf{q}}^{\dagger} e^{-i \mathbf{q} \cdot \boldsymbol{\rho}}\right) \\
& \times \sin \frac{n \pi z}{d} \theta\left(\omega_{D}-\omega_{n q}\right),
\end{aligned}
$$

where $\omega_{D}$ is the Debye frequency. With the foregoing, the electron-phonon interaction Hamiltonian becomes

$$
\begin{aligned}
\hat{H}_{\mathrm{el}-\mathrm{ph}}= & \frac{z e^{2} n_{0}}{c \sqrt{\varrho_{0}}} \sum_{n \mathbf{q}}\left(\frac{\hbar \omega_{n q}}{2 V}\right)^{1 / 2} \theta\left(\omega_{D}-\omega_{n q}\right) \sum_{m m^{\prime}} \sum_{\mathbf{p} \sigma} \frac{1}{\pi} \\
& \times \sum_{l} d_{m m^{\prime} l} v_{l n \mathbf{q}}^{\mathrm{scr}}\left(b_{n \mathbf{q}} c_{m \mathbf{p} \sigma}^{\dagger} c_{m^{\prime} \mathbf{p}-\mathbf{q} \sigma}+b_{n \mathbf{q}}^{\dagger} c_{m \mathbf{p} \sigma}^{\dagger} c_{m^{\prime} \mathbf{p}+\mathbf{q} \sigma}\right) .
\end{aligned}
$$

In the above,

$$
\begin{aligned}
d_{m m^{\prime} l} & =\frac{2 \pi}{d} \int_{0}^{d} d z \sin \frac{m \pi z}{d} \sin \frac{m^{\prime} \pi z}{d} \sin \frac{l \pi z}{d} \\
& =\frac{\left[(-1)^{m+m^{\prime}+l}-1\right] 4 m m^{\prime} l}{m^{4}+m^{\prime 4}+l^{4}-2\left(m^{2} m^{\prime 2}+m^{\prime 2} l^{2}+l^{2} m^{2}\right)} .
\end{aligned}
$$

To derive the form of the phonon-mediated attractive interaction, it is convenient to rewrite $\hat{H}_{\mathrm{el}-\mathrm{ph}}$ in terms of the electron and phonon fields. The latter is given by

$$
\begin{aligned}
\hat{\varphi}(\mathbf{r})= & \sum_{n} \varphi_{n}(\mathbf{r}) \\
\equiv & \sum_{n} \sum_{\mathbf{q}}\left(\frac{\hbar \omega_{n q}}{2 V}\right)^{1 / 2} \theta\left(\omega_{D}-\omega_{n q}\right) \\
& \times\left(b_{n \mathbf{q}} e^{i \mathbf{q} \cdot \boldsymbol{\rho}}+b_{n \mathbf{q}}^{\dagger} e^{-i \mathbf{q} \cdot \boldsymbol{\rho}}\right) \sin \frac{n \pi z}{d},
\end{aligned}
$$

where $\varphi_{n}$ is the $n$ th, confinement induced, branch of the phonon field. As discussed in Sec. III B below, in the $\mathbf{q} \rightarrow 0$ limit, the screened Coulomb potential matrix in Eq. (14) is dominated by its diagonal terms. Thus making the approximation $v_{\ln \mathbf{q} \rightarrow 0}^{\mathrm{scr}}=$ $\delta_{l n} v_{n n \mathbf{q} \rightarrow 0}^{\mathrm{scr}}$, the electron-phonon Hamiltonian can be rewritten

$$
\hat{H}_{\mathrm{el}-\mathrm{ph}}=\sum_{n} \gamma_{n} \int d^{3} r \sum_{\sigma} \hat{\psi}_{\sigma}^{\dagger}(\mathbf{r}) \hat{\psi}_{\sigma}(\mathbf{r}) \hat{\varphi}_{n}(\mathbf{r}),
$$

with electron-phonon coupling constant

$$
\gamma_{n}=\left(\frac{z e^{2} n_{0}}{c \sqrt{\varrho_{0}}}\right) v_{n n \mathbf{q} \rightarrow 0}^{\mathrm{scr}}
$$

for phonon branch $n$. We now turn our attention to the screened Coulomb potential and to its size dependence.

\section{B. Screened Coulomb interaction}

To determine the form of the screened Coulomb interaction in a nanofilm, we must calculate the dielectric function. For this, we follow the self-consistent field method and perform the calculation in the random phase approximation (RPA). ${ }^{29}$ A similar study has been carried out before, focusing on the plasmon dispersion relation. ${ }^{33}$ Here, we are interested in the static, long-wavelength limit. In the derivation below, we emphasize the most important points and give the more technical details in Appendix A.

\section{Dielectric function and screening of the Coulomb potential}

Let $V^{\text {ext }}$ denote the potential due to an external charge, or impurity, in the system. Within linear response, the total potential, $V=V^{\text {ext }}+V^{\text {scr }}$, where $V^{\text {scr }}$ is the screening, or induced potential, is related to the external potential by the dielectric function

$$
V^{\mathrm{ext}}(\mathbf{r}, t)=\int d^{3} r^{\prime} d t^{\prime} \varepsilon\left(\mathbf{r}, \mathbf{r}^{\prime}, t-t^{\prime}\right) V\left(\mathbf{r}^{\prime}, t^{\prime}\right),
$$

or $V^{\text {ext }}(\mathbf{r}, \omega)=\int d^{3} r^{\prime} \varepsilon\left(\mathbf{r}, \mathbf{r}^{\prime}, \omega\right) V\left(\mathbf{r}^{\prime}, \omega\right)$ in frequency domain. Exploiting the translational invariance of the system in the $x O y$ plane, we expand the involved quantities over our singleelectron basis. This yields

$$
V_{n \mathbf{q}}^{\mathrm{ext}}(\omega)=\sum_{m} \varepsilon_{n m \mathbf{q}}(\omega) V_{m \mathbf{q}}(\omega) .
$$

On the other hand, the external potential is given by

$$
V^{\operatorname{ext}}(\mathbf{r}, \omega)=\int d^{3} r^{\prime} \frac{\varrho^{\operatorname{ext}}\left(\mathbf{r}^{\prime}, \omega\right)}{\left|\mathbf{r}-\mathbf{r}^{\prime}\right|},
$$

or, equivalently,

$$
V_{n \mathbf{q}}^{\mathrm{ext}}(\omega)=\sum_{m} v_{n m \mathbf{q}} \varrho_{m \mathbf{q}}^{\mathrm{ext}}(\omega) .
$$

The expansion coefficients of the (bare) Coulomb potential $v_{n m \mathbf{q}}$ are given by Eq. (A7) in Appendix A. Equating Eqs. (20) and (22) above, one has

$$
\begin{aligned}
V_{m \mathbf{q}}(\omega) & =\sum_{n} \varepsilon_{m n \mathbf{q}}^{-1}(\omega) V_{n \mathbf{q}}^{\mathrm{ext}}(\omega) \\
& =\sum_{n^{\prime}} v_{m n^{\prime} \mathbf{q}}^{\mathrm{scr}}(\omega) \varrho_{n^{\prime} \mathbf{q}}^{\mathrm{ext}}(\omega),
\end{aligned}
$$

with the screened Coulomb potential given by

$$
v_{m n^{\prime} \mathbf{q}}^{\mathrm{scr}}(\omega)=\sum_{n} \varepsilon_{m n \mathbf{q}}^{-1}(\omega) v_{n n^{\prime} \mathbf{q}} .
$$

\section{Dielectric function in the RPA}

Our treatment here is similar to that of Mahan for the three-dimensional electron gas. ${ }^{29}$ We consider the Heisenberg equation for the (electron) particle density operator,

$$
-i \hbar \frac{\partial}{\partial t} \hat{n}_{n \mathbf{q}}=\left[\hat{H}, \hat{n}_{n \mathbf{q}}\right] \text {. }
$$

where $\hat{n}_{n \mathbf{q}}=\int d^{3} r \psi_{n \mathbf{q}}^{*}(\mathbf{r}) \hat{n}(\mathbf{r})$, with $\hat{n}(\mathbf{r})=\sum_{\sigma} \hat{\psi}_{\sigma}^{\dagger}(\mathbf{r}) \hat{\psi}_{\sigma}(\mathbf{r})$. The Hamiltonian above is $\hat{H}=\hat{H}_{0}+\hat{V}$. The noninteracting Hamiltonian is $\hat{H}_{0}=\sum_{n \mathbf{q} \sigma} E_{n \mathbf{q}} c_{n \mathbf{q} \sigma}^{\dagger} c_{n \mathbf{q} \sigma}$. The interacting term is given by the total potential operator, $\hat{V}$ (this is the principal assumption of the self-consistent field method). ${ }^{29}$ In terms of the particle density operator, it is written

$$
\begin{aligned}
\hat{V} & =-e \sum_{\sigma} \int d^{3} r \hat{\psi}_{\sigma}^{\dagger}(\mathbf{r}) V(\mathbf{r}) \hat{\psi}_{\sigma}(\mathbf{r}) \\
& =-e \sum_{n \mathbf{q}} \hat{n}_{n-\mathbf{q}} V_{n \mathbf{q}} .
\end{aligned}
$$


As shown in Appendix A, solving the equation of motion for the particle density within the RPA allows one to write the induced charge density, $\varrho_{n \mathbf{q}}(\omega)=-e\left\langle\hat{n}_{n \mathbf{q}}(\omega)\right\rangle$, in terms of the polarization and the total potential as

$$
\varrho_{n \mathbf{q}}(\omega)=e^{2} \sum_{l} P_{n l \mathbf{q}}^{(1)}(\omega) V_{l \mathbf{q}}(\omega)
$$

The polarization is given by

$$
P_{n l \mathbf{q}}^{(1)}(\omega)=\frac{2}{\pi^{2} L^{2} d} \sum_{m m^{\prime}} d_{n m m^{\prime}} d_{l m m^{\prime}} \sum_{\mathbf{p}} \frac{n_{\mathrm{F}}\left(\epsilon_{m \mathbf{p}}\right)-n_{\mathrm{F}}\left(\epsilon_{m^{\prime} \mathbf{p}+\mathbf{q}}\right)}{\hbar \omega-\epsilon_{m^{\prime} \mathbf{p}+\mathbf{q}}+\epsilon_{m \mathbf{p}}},
$$

where the $\epsilon_{m \mathbf{p}}=E_{m \mathbf{p}}-\mu$ are the independent electron energies measured with respect to the Fermi level and $n_{\mathrm{F}}$ is the Fermi occupation function $\left[n_{\mathrm{F}}(\epsilon)=1\right.$ if $\epsilon \leqslant 0$, and 0 otherwise]. Now, given that the induced charge density gives rise to the screening potential $V_{n \mathbf{q}}^{\mathrm{scr}}(\omega)=\sum_{m} v_{n m \mathbf{q}} \varrho_{m \mathbf{q}}(\omega)$ and that $V_{n \mathbf{q}}^{\mathrm{ext}}(\omega)=V_{n \mathbf{q}}(\omega)-V_{n \mathbf{q}}^{\mathrm{scr}}(\omega)$, one has

$$
V_{n \mathbf{q}}^{\mathrm{ext}}(\omega)=V_{n \mathbf{q}}(\omega)-e^{2} \sum_{m l} v_{n m \mathbf{q}} P_{m l \mathbf{q}}^{(1)}(\omega) V_{l \mathbf{q}}(\omega)
$$

Thus, from Eq. (20), we finally have the dielectric function

$$
\varepsilon_{n l \mathbf{q}}(\omega)=\delta_{n l}-e^{2} \sum_{m} v_{n m \mathbf{q}} P_{m l \mathbf{q}}^{(1)}(\omega) .
$$

To determine the long-wavelength limit of the static, screened Coulomb potential, $v_{m n^{\prime} \mathbf{q} \rightarrow 0}^{\mathrm{scr}}(\omega=0)$ [cf. Eq (24)], we have to evaluate $\lim _{q \rightarrow 0} \sum_{n} \varepsilon_{m n \mathbf{q}}^{-1}(0) v_{n n^{\prime} \mathbf{q}}$. The longwavelength limit of the static dielectric function, $\varepsilon_{m n \mathbf{q} \rightarrow 0}(0)$, can be obtained in closed form (see Appendix A). However, to obtain a converged result for the nanofilm thicknesses considered in this study requires including up to 600 bands in the calculation of the polarization $P^{(1)}$. We found that $200 \times 200$ matrices have to be considered in the calculation of $v^{\text {scr }}=\varepsilon^{-1} v$ for the matrix elements of $v^{\text {scr }}$ to be converged. Thus $v_{m n^{\prime} \mathbf{q} \rightarrow 0}^{\mathrm{scr}}(0)$ is evaluated numerically. The calculations show that $v_{m n^{\prime} \mathbf{q} \rightarrow 0}^{\text {scr }}$ is dominated by its diagonal terms. Hence, to a first approximation, we take $v_{n n^{\prime} \mathbf{q} \rightarrow 0}^{\text {scr }} \simeq \delta_{n n^{\prime}} v_{n n \mathbf{q} \rightarrow 0}^{\mathrm{scr}}$.

For later discussion, we point out that the product $d_{n m m^{\prime}} d_{l m m^{\prime}}$ in the polarization in Eq. (28) implies that its matrix elements are nonzero only if the indices $n$ and $l$ are both of the same parity [cf. Eq. (15)]. As discussed in Appendix A, the bare Coulomb potential shares a similar property and, furthermore, in the long-wavelength limit $\mathbf{q} \rightarrow 0$, it is largely governed by its matrix elements with odd indices. Thus, in that limit, the dielectric function matrix elements in Eq. (30) with even indices will have comparatively little contribution from the polarization.

\section{Effective electron-electron interaction}

To obtain the phonon-exchange mediated effective electron-electron interaction, we first define the phonon Green functions

$$
i D_{n m}^{0}\left(\mathbf{r}, t ; \mathbf{r}^{\prime}, t^{\prime}\right)=\left\langle T\left[\hat{\varphi}_{n}(\mathbf{r}, t) \hat{\varphi}_{m}\left(\mathbf{r}^{\prime}, t^{\prime}\right)\right]\right\rangle .
$$

Because of the invariance with respect to translation in time, $D^{0}$ depends only on $t-t^{\prime}$. Given the phonon field expansion in Eq. (16), one shows

$$
D_{n m}^{0}\left(\mathbf{r}, \mathbf{r}^{\prime}, \omega\right)=\delta_{n m} \sum_{\mathbf{q}} \psi_{n \mathbf{q}}(\mathbf{r}) \psi_{n \mathbf{q}}^{*}\left(\mathbf{r}^{\prime}\right) D_{n}^{0}(n \mathbf{q}, \omega),
$$

with

$$
D_{n}^{0}(n \mathbf{q}, \omega)=\hbar \frac{\omega_{n \mathbf{q}}^{2}}{\omega^{2}-\left(\omega_{n \mathbf{q}}-i \eta\right)^{2}} \theta\left(\omega_{D}-\omega_{n \mathbf{q}}\right) .
$$

As in the three-dimensional case, ${ }^{28}$ there is a one-to-one correspondence between the phonon-exchange contributions to the electron Green function and those of a spin-independent interaction. For instance, the lowest phonon-exchange contribution to the electron Green function is

$$
\begin{aligned}
i G_{\sigma}^{(2)}\left(\mathbf{r}, t ; \mathbf{r}^{\prime} t^{\prime}\right)= & \left(\frac{-i}{\hbar}\right)^{2} \frac{1}{2 !} \int d t_{1} d t_{2}\left\langleT \left[\hat{H}_{\mathrm{el}-\mathrm{ph}}\left(t_{1}\right)\right.\right. \\
& \left.\left.\times \hat{H}_{\mathrm{el}-\mathrm{ph}}\left(t_{2}\right) \hat{\psi}_{\sigma}(\mathbf{r}, t) \hat{\psi}_{\sigma}^{\dagger}\left(\mathbf{r}^{\prime}, t^{\prime}\right)\right]\right\rangle_{\mathrm{conn} .},
\end{aligned}
$$

where only connected diagrams need to be considered. Expanding the electron and phonon fields in terms of the corresponding creation and destruction operators, allows one to show that the above is equivalent to the first-order contribution to the electron Green function of a frequency dependent, effective interaction $v^{\text {eff }}$. The derivation is very similar to the one in the three-dimensional case with the only difference that we have an additional quantum number due to confinement. The expansion coefficients of the effective interaction are found to be

$$
v_{n n^{\prime} \mathbf{p}}^{\mathrm{eff}}(\omega)=\frac{1}{\hbar} \delta_{n n^{\prime}} D_{n}^{0}(n \mathbf{p}, \omega) \gamma_{n}^{2} .
$$

Then, in the static limit [cf. Eq. (33)], one has

$$
v_{n n^{\prime} \mathbf{p}}^{\mathrm{eff}}(\omega \rightarrow 0)=-\delta_{n n^{\prime}} \gamma_{n}^{2} \theta\left(\omega_{D}-\omega_{n \mathbf{p}}\right),
$$

so it is an attractive interaction. Assuming $\omega_{D} \gg \omega_{n \mathbf{p}}$, one has $v_{n n^{\prime} \mathbf{p}}^{\text {eff }}(0)=-\delta_{n n^{\prime}} \gamma_{n}^{2}$. In real space, the equivalent, effective interaction is readily calculated to be

$v_{\mathrm{eff}}\left(\mathbf{r}, \mathbf{r}^{\prime}\right)=-\frac{2}{d} \delta\left(\boldsymbol{\rho}-\boldsymbol{\rho}^{\prime}\right) \sum_{n} \gamma_{n}^{2} \sin \frac{2 \pi n z}{d} \sin \frac{2 \pi n z^{\prime}}{d}$.

Note that in the $d \rightarrow \infty$ limit (bulk limit), one can verify that the different electron-phonon coupling constants tend to the same value, $\gamma_{n} \rightarrow \gamma$. Thus, in that limit, the present approximation correctly reduces to an effective interaction given by an attractive delta function, or contact potential $v_{\text {eff }}\left(\mathbf{r}, \mathbf{r}^{\prime}\right)=-\gamma^{2} \delta\left(\mathbf{r}-\mathbf{r}^{\prime}\right),{ }^{34}$ which is the form commonly used in the three-dimensional case. ${ }^{28,29}$

\section{CRITICAL PARAMETERS OF A NANOFILM}

To determine the critical parameters in a nanofilm, we rewrite the gap equation in terms of the above result. First, the effective interaction matrix elements between pairs of timereversed states are given by $\left\langle\mathbf{q} n,-\mathbf{q} n\left|v_{\text {eff }}\right| \mathbf{q}^{\prime} n^{\prime},-\mathbf{q}^{\prime} n^{\prime}\right\rangle=$ $U_{n n^{\prime}} / L^{2} d$ with

$$
U_{n n^{\prime}}=2 \sum_{m} \gamma_{m}^{2} d_{m n n^{\prime}}^{2} / \pi^{2}
$$

and the $d_{m n n^{\prime}}$ are given in Eq. (15). Note that the summation over $m$ above is finite. Indeed, in single-crystal nanofilms, 
the number of phonon modes contributing to the effective interaction is given by the number of monolayers in the film. ${ }^{32,35}$ Second, we assume that the phonon mediated attractive interaction is effective only between states within a thin energy window of width $2 \hbar \omega_{D}$ around the Fermi level, $E_{F}$. Hence we finally write

$$
V_{\mathbf{q} n, \mathbf{q}^{\prime} n^{\prime}}=\frac{U_{n n^{\prime}}}{L^{2} d} \theta\left(\hbar \omega_{D}-\left|\epsilon_{\mathbf{q} n}\right|\right) \theta\left(\hbar \omega_{D}-\left|\epsilon_{\mathbf{q}^{\prime} n^{\prime}}\right|\right) .
$$

We introduced this expression in the gap equation (8). The above implies that $\Delta(\mathbf{q} n)$ vanishes for states outside the mentioned window. Furthermore, within that window, the energy gap depends only on the band index. Hence, for short, we introduce $\Delta_{n}=\Delta(\mathbf{q} n)$. The summation over momentum in Eq. (8) can be transformed into an integral over energy, weighted by the electron density of states, leading finally to the multigap equation

$$
\Delta_{n}=\sum_{n^{\prime}} U_{n n^{\prime}} \Delta_{n^{\prime}} \int_{-\hbar \omega_{D}}^{+\hbar \omega_{D}} d \epsilon \mathcal{N}_{n^{\prime}}(\epsilon) \frac{1}{2 \xi_{n^{\prime}}} \tanh \left(\frac{\xi_{n^{\prime}}}{2 k_{\mathrm{B}} T}\right) .
$$

In the above, $\mathcal{N}_{n}(\epsilon)=\theta\left(\epsilon-\hbar^{2} n^{2} \pi^{2} / 2 m d^{2}+\mu\right) / 4 \pi d$ is the $n$th band density of states per spin. The number of gap values is given by the number of occupied bands. The degeneracy or not of the gap values above is determined by the $U_{n n^{\prime}}$, i.e., by the form of $v_{\text {eff }}$. For instance, in the case of the models of Refs. 2 and 6, which use a contact potential, the gap values are all degenerate. Here, phonon confinement modifies the contact interaction in such a way that the gap values are typically nondegenerate. ${ }^{36}$

Before studying the solutions of the gap equation, a note on the parameter values used is in order. For definiteness, we choose parameter values corresponding to Al. Hence $r_{s}=$ $2.07 a_{0}$ gives the particle density, ${ }^{37}$ and $\hbar \omega_{D} / k_{B}=375 \mathrm{~K}^{28}$ For reference, the bulk critical parameters of $\mathrm{Al}$ are $T_{c}^{b}=$ $1.2 \mathrm{~K}$ and $\Delta^{b}=1.7 k_{B} T_{c}^{b} \cdot{ }^{37}$ For the term in parentheses in the electron-phonon coupling constants (18), one could use the bulk value. However, there are multiple reports indicating that the sound velocity in nanofilms can be strongly reduced compared to its bulk counterpart. ${ }^{38-40}$ Thus the value used has to be rescaled accordingly. For instance, in $\mathrm{Cu}$ nanofilms 20-, $10-$, and 4-nm thick, the sound velocity was measured to be $14 \%, 20 \%$, and $38 \%$ lower than in the bulk. ${ }^{40}$ Unfortunately, directly measured values for $\mathrm{Al}$ nanofilms are not available. Still, one can make an estimate based on the average gap value reported by Court et al. for Al nanofilms 5-nm thick, which are the thinnest nanofilms they studied. ${ }^{14}$ We find that in our model, the gap value reported implies a sound velocity $30 \%$ lower than in the bulk. We use this value for our analysis of films a fraction to a few nanometers thick. As will be clear further down, the qualitative conclusions of our study do not depend on this choice. ${ }^{41,42}$

The gap equation is a nonlinear equation. To solve it [cf. Eq. (40)] and for all our calculations in this work, we use MATLAB. ${ }^{43}$ The energy gaps depend on temperature, vanishing at the critical temperature, $T_{c}$. This is shown in Fig. 1(a) for a nanofilm with thickness $d=13.3 a_{0}$. In this case, there are four occupied bands, resulting in four energy gap values. The critical temperature is $T_{c}=2.8 T_{c}^{b}$. At $T=0$, the energy gaps are also larger than the bulk energy gap (the values at $T=0$ are
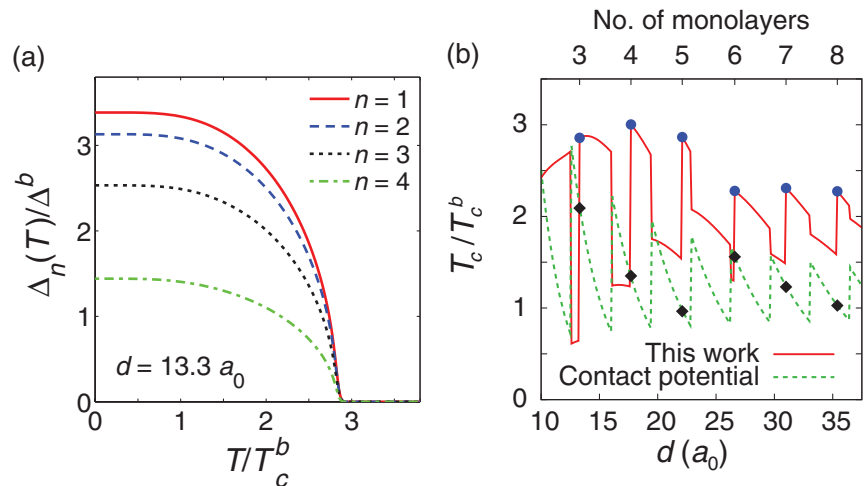

FIG. 1. (Color online) (a) Temperature dependence of the four gap values of a $13.3 a_{0} \mathrm{Al}$ nanofilm [three (111) monolayers thick] The critical temperature is $T_{c}=2.8 T_{c}^{b}$. (b) Dependence of $T_{c}$ on film thickness. Confinement effects on the coupled electron and phonon systems have a strong impact (solid line); as $d$ increases, $T_{c}$ drops when a new electron band starts to be occupied but rises when the number of phonon modes (given by the number of monolayers) contributing to the pairing increases. The filled circles indicate the predicted $T_{c}$ as a function of the number of monolayers in the film (cf. upper scale). In the contact-potential case (dotted line), $T_{c}$ depends on $d$ only through the total electron density of states per spin at the Fermi level. The diamonds indicate the $T_{c}$ as a function on number of monolayers in the contact-potential case.

$\Delta_{1}=3.4, \Delta_{2}=3.1, \Delta_{3}=2.5$, and $\Delta_{4}=1.4$, in units of the bulk value, $\Delta^{b}$ ). To determine the number of phonon modes contributing to electron-phonon coupling [cf. Eq. (38)], we assumed a film grown in the [111] direction, which is a typical direction in previous works on $\mathrm{Pb}$ nanofilms ${ }^{12,19}$ and on $\mathrm{Al}$ nanofilms. ${ }^{18}$ With a lattice constant of $4.05 \AA$ for $\mathrm{Al}$, three phonon modes contribute to electron-phonon coupling in the present case.

Turning to the dependence of the critical parameters on nanofilm thickness, in Fig. 1(b), we show the behavior of $T_{c}$ as $d$ is varied continuously (solid line). As above, for this calculation, we assume a film grown in the [111] direction. In experiment, the nanofilm thickness is not a continuous variable, but is given by the number of monolayers deposited. Thus more relevant to experiment are the predicted $T_{c}$ 's for the latter. These are indicated by the filled circles in the figure. The full effects of confinement are best discussed by making a comparison with the $T_{c}$ 's obtained using in our calculations a contact potential, i.e., $v_{\text {eff }}\left(\mathbf{r}, \mathbf{r}^{\prime}\right)=-g \delta\left(\mathbf{r}-\mathbf{r}^{\prime}\right)$, instead of Eq. (37), with a $g$ value obtained from experimental data as in, e.g., Ref. 15. The resulting $T_{c}$ 's as a function of $d$ (we note that they are very close to those in Ref. 15), are given by the dotted line in Fig. 1(b) with the diamonds indicating the values as a function of the number of (111) monolayers. As mentioned in Introduction, the sawtooth pattern observed in this approach as $d$ varies continuously was shown to arise because the total electron density of states per spin at the Fermi level, $\mathcal{N}(0)=\sum_{n} \mathcal{N}_{n}(0)$, increases suddenly when a new band starts to be occupied as $d$ increases and then decreases monotonically $\left[\mathcal{N}_{n}(0) \propto 1 / d\right.$, cf. the equation above] until the band above starts to be occupied in turn. ${ }^{2,6,15}$

Our results are in stark contrast with the above. We find, remarkably, that in ultrathin nanofilms, $T_{c}$ falls instead 
(a)

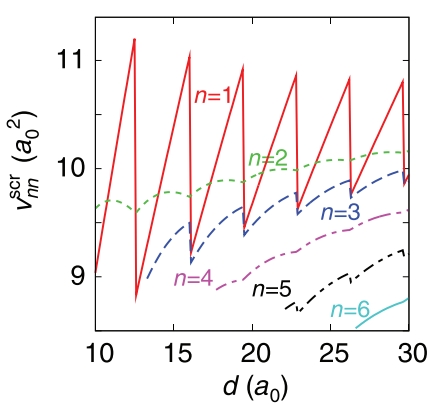

(b)

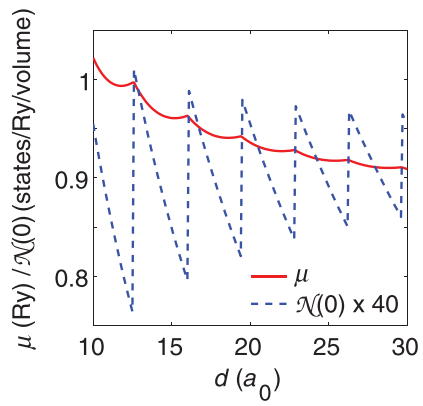

FIG. 2. (Color online) (a) Diagonal elements of the longwavelength limit of the static screened Coulomb interaction, $v^{\text {scr }}=$ $\varepsilon^{-1} v$, as a function of film thickness. Sharp drops occur whenever the number of occupied bands increases. The drops are due to the resulting increase of screening in the nanofilm. (b) Fermi level $\mu$ (solid line) and total electron density of states per spin at the Fermi level, $\mathcal{N}(0)=\sum_{n} \mathcal{N}_{n}(0)$, as a function of $d$ (dashed line). The kinks in $\mu$ and sharp peaks in $\mathcal{N}(0)$ indicate when a new band starts to be occupied as $d$ increases.

of increasing when the number of occupied electron bands increases by one, but rises when the number of phonon modes increases by one. This can be readily understood as follows. On the one hand, there is the dependence of the electronphonon coupling constants $\gamma_{n}$ on the nanofilm thickness. As shown in the previous section, $\gamma_{n}$ is a constant times $v_{n n \mathbf{q} \rightarrow 0}^{\mathrm{scr}}$. Thus, in Fig. 2(a), we plot the latter as a function of $d$. One can see that the matrix elements of the screened Coulomb interaction show strong oscillations with varying $d$, dropping clearly whenever a new band starts to be occupied [compare with the dotted line in Fig. 1(b)], particularly for symmetric states ( $n$ odd). The direct correlation with band occupation is made evident by Fig. 2(b), where we plot $\mathcal{N}(0)$ as a function of $d$. The fact that the screened Coulomb interaction drops when the number of occupied bands increases is due to the fact that the polarization and the dielectric function depends on the number of occupied bands [cf. Eqs. (28) and (30)]. When the number of occupied bands increases, the polarization increases as well, leading to a stronger screening. This results in the drop of $v_{n n \mathbf{q} \rightarrow 0}^{\mathrm{scr}}$ and a weaker electron-phonon coupling. ${ }^{44}$ This effect was already discussed by Mahan, in a work addressing electron-phonon coupling near van Hove singularities. ${ }^{45}$ The weaker electron-phonon coupling leads to a lowering of $T_{c}$. On the other hand, the number of standing phonon modes contributing to the electron-phonon coupling increases whenever a new monolayer is added to a crystal film, ${ }^{32}$ thereby increasing the strength of the effective pairing interaction [cf. Eq. (38)] and causing $T_{c}$ to suddenly increase. It is, hence, the interplay between the two effects that determines $T_{c}$. In Fig. 1(b), one can see that the resulting $T_{c}$ 's are larger than the bulk $T_{c}$. This is in line with the reports mentioned above ${ }^{14,18}$ in which confinement effects in Al nanofilms were found to enhance the superconducting critical parameters with respect to the bulk values.

Implicit in the above is that the behavior of $T_{c}$ with varying $d$ can depend on the direction of growth of the nanofilm. Indeed, the number and the properties of the confined phonon modes involved are determined by the latter. This is in line
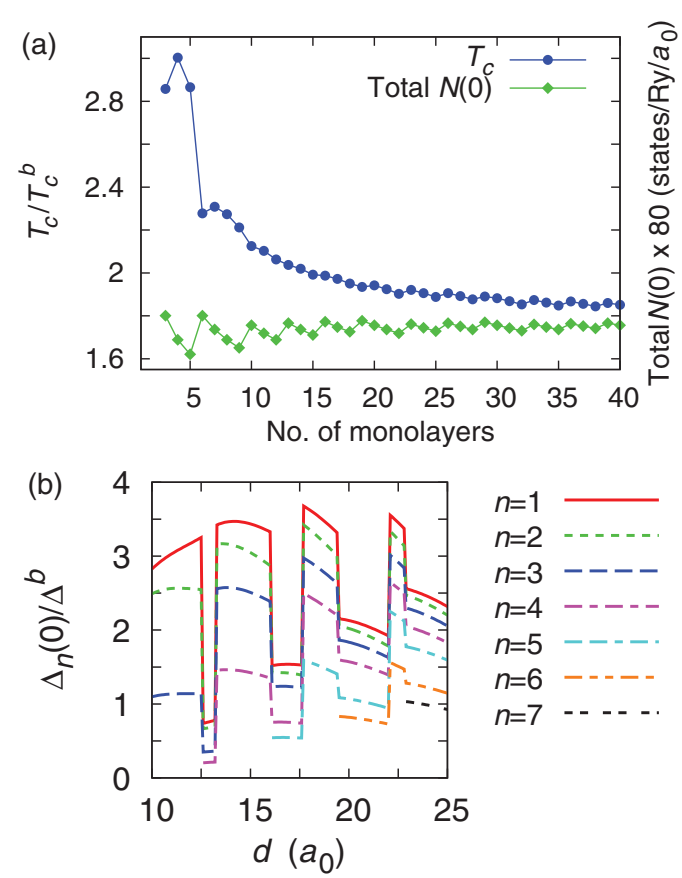

FIG. 3. (Color online) (a) Dependence of $T_{c}$ and of $\mathcal{N}(0)$ on the number of (111) monolayers. Only for films thicker than a critical number of monolayers do $T_{c}$ and the total density of states correlate. In the present example, this occurs above $\sim 20$ monolayers. (b) The dependence of the energy gap values on $d$. As $d$ increases, a new gap value appears as a new band is occupied, i.e., a new subcondensate is formed (cf. Sec. V). The trends in the dependence on $d$ are similar to those of $T_{c}$ [compare with Fig. 1(b)]. The spread in gap values rapidly decreases with film thickness.

with the results in Ref. 18, where an estimation of $T_{c}$ is done based on a density functional theory calculation of electron-phonon coupling in $\mathrm{Al}$ nanofilms. Indeed, it is found that the dependence of $T_{c}$ on the number of monolayers in the [111] direction is different from that in the [100] direction. Furthermore, in the latter case, no correlation is found between $T_{c}$ and $\mathcal{N}(0){ }^{18}$ A reasonable level of correlation is found, however, for films grown in the [111] direction. In our model, this occurs only at higher film thicknesses, when the change in electron-phonon coupling with the increase of occupied bands becomes sufficiently weak. This is shown in Fig. 3(a), where we plot $T_{c}$ and $\mathcal{N}(0)$ as a function of the number of (111) monolayers up to a thickness of 40 monolayers. One can see that the two quantities start following the same pattern for thicknesses above $\sim 20$ monolayers, as the confined electronic states assume a dominant role. A more accurate calculation of this threshold number of monolayers requires going beyond the approximations of the phonon and electron-phonon interaction models used here.

Consider now the gap dependence on nanofilm thickness. We show this in Fig. 3(b). As $d$ increases, a new energy gap value appears each time that a new electron energy band starts to be occupied. As one can expect, the dependence of the gap values on $d$ mirrors that of $T_{c}$. One can also observe that the spread of gap values decreases with increasing $d$. This can be understood from the following. As indicated in the discussion following Eq. (37), for $d \rightarrow \infty$, the electron-phonon coupling 
constants tend to a single value $\gamma$, and $v_{\text {eff }}$ becomes the three-dimensional contact potential. Assuming the bulk value for the speed of sound, $\gamma$ takes its bulk value and so does the energy gap. ${ }^{46}$ We believe that the different gap values should be experimentally discernible in nanofilms up to a few monolayers thick. For thicker nanofilms, this is probably difficult because the difference between gap values becomes too small, although some effects due to the multiple gaps might still be observable. In this regard, it is interesting that, in their differential conductance measurements on 5-nm thick Al nanofilms, Court et al. observe subgap structure, below a main peak at $2 \Delta=0.608 \mathrm{meV} .{ }^{14}$ One must bare in mind, however, that in those experiments, the samples are polycrystalline, so the effects of phonon confinement are more difficult to ascertain than in single crystal samples.

Increases in the critical parameters as the number of phonon modes contributing to electron-phonon coupling increases with nanofilm thickness were already obtained by Hwang and co-workers in Ref. 7 (to our knowledge, the first work to address the effects of phonon confinement on superconductivity). In that work, however, the effect of confinement on the strength of the electron-phonon coupling and, hence, on the strength of the effective pairing interaction, is not taken into account. Hence the model in Ref. 7 is insufficient to observe the drops in the critical parameters when the number of occupied electron bands increases. Furthermore, the increase in the critical parameters that they find with the increase of the number of phonon modes is, compared to what we find, rather weak. This is probably due to their use of a bulk value for the deformation potential coupling constant, ${ }^{7}$ since that is equivalent to using the bulk value for the sound velocity in our formulation.

\section{CONDENSATE WAVE FUNCTION}

We now turn to the effects of confinement on the condensate, or Cooper pair, wave function, defined by $\Psi\left(\mathbf{r} \mathbf{r}^{\prime}\right)=\mathcal{F}\left(\mathbf{r} \mathbf{r}^{\prime}, 0\right)$, with $\mathcal{F}$ the Gor'kov function ${ }^{47}$ (also called "anomalous average") introduced in Sec. II above. Thus, in terms of the single-electron basis, one has $\Psi\left(\mathbf{r} \mathbf{r}^{\prime}\right)=$ $\sum_{n} \psi_{\mathbf{q} n}(\mathbf{r}) \psi_{-\mathbf{q} n}\left(\mathbf{r}^{\prime}\right) \mathcal{F}(\mathbf{q} n,-\mathbf{q} n, 0)$. As in the derivation of the gap equation, we exploit the fact that $\mathcal{F}(\mathbf{q} n,-\mathbf{q} n, 0)=$ $\frac{1}{\beta \hbar} \sum_{p} \tilde{\mathcal{F}}\left(\mathbf{q} n,-\mathbf{q} n, i \omega_{p}\right)$. Then, it is readily found that at
$T=0 \mathrm{~K}$, one has

$$
\Psi\left(\mathbf{r} \mathbf{r}^{\prime}\right)=\sum_{\mathbf{q} n} \psi_{\mathbf{q} n}(\mathbf{r}) \psi_{-\mathbf{q} n}\left(\mathbf{r}^{\prime}\right) \Delta_{n} \frac{1}{2 \xi_{\mathbf{q} n}} .
$$

To better analyze the wave function, it is convenient to rewrite it in terms of the center of mass and relative coordinates: $\left(\rho, z ; \rho^{\prime}, z^{\prime}\right) \rightarrow(\mathbf{R}, Z ; \mathbf{u}, \zeta)$, with $\mathbf{R}=\left(\rho+\rho^{\prime}\right) / 2$, $Z=\left(z+z^{\prime}\right) / 2$, and $\mathbf{u}=\rho-\boldsymbol{\rho}^{\prime}, \zeta=z-z^{\prime}$. The dependence on the former reflects the inhomogeneity of the system, while the symmetry of the pairing is associated with the latter. Because of the translational symmetry in the $x O y$ plane, the pair wave function does not depend on $\mathbf{R}$, and because of rotational symmetry, the dependence on ureduces to a dependence on $u=\|\mathbf{u}\|$. From Eq. (41), transforming the summation over $\mathbf{k}$ into an integration, one has

$$
\begin{aligned}
\Psi & =\Psi(Z ; u, \zeta)=\sum_{n} \Psi_{n}(Z ; u, \zeta) \\
& =\frac{1}{4 \pi^{2} d} \sum_{n} \Delta_{n} \sin \frac{n \pi\left(Z+\frac{\zeta}{2}\right)}{d} \sin \frac{n \pi\left(Z-\frac{\zeta}{2}\right)}{d} I_{n}(u),
\end{aligned}
$$

with

$$
I_{n}(u)=\int_{k_{\min }^{(n)}}^{k_{\max }^{(n)}} d k k \frac{J_{0}(k u)}{\sqrt{\epsilon_{k n}^{2}+\Delta_{n}^{2}}} .
$$

The $\left(k_{\min }^{(n)}, k_{\max }^{(n)}\right)$ range accounts for the fact that the gap values vanish for momenta outside the energy window defined by $\hbar \omega_{D}$. The pair wave function is thus a local quantity, in the sense that it does not only depend on the relative coordinates of the paired particles but also on the position of their center of mass.

In Fig. 4, we plot the Cooper pair wave function probability density as a function of the relative coordinate $\mathbf{u}$, around the center of mass coordinates, for $Z=d / 2$ and $\zeta=d / 2$, with $d \simeq 13.3 a_{0}$, i.e., an $N=3$ (111) monolayers thick nanofilm. The oscillations as a function of $u$ can be expected from the qualitative behavior of the Cooper pair wave function in homogeneous systems. ${ }^{48}$ Interestingly, however, in the present case, the amplitude of the oscillations is modulated. The amplitude decreases distinctly in the vicinity of the

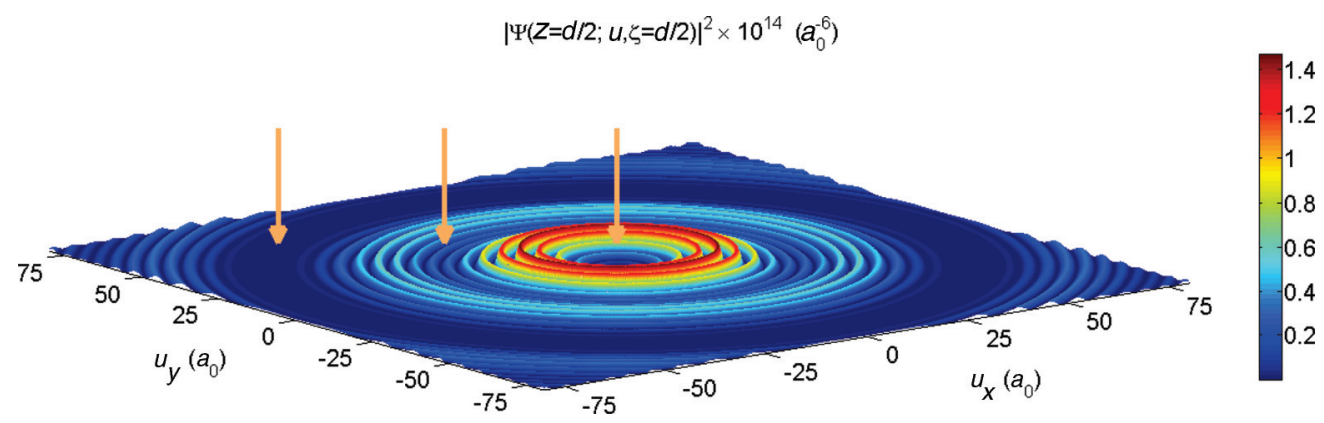

FIG. 4. (Color online) Typical dependence of the Cooper pair wave-function probability density on the relative coordinate $\mathbf{u}$. The plot is for $Z=d / 2$ and $\zeta=d / 2$, with $d=13.3 a_{0}$. The amplitude of the oscillations as a function of interparticle distance $u$ is clearly modulated. The condensate tends to be depleted of Cooper pairs for interparticle distances close to those indicated by the vertical arrows. The condensate being composed by distinct subcondensates, the regions of low amplitude are due to interference between the wave functions associated with each of the subcondensates. 

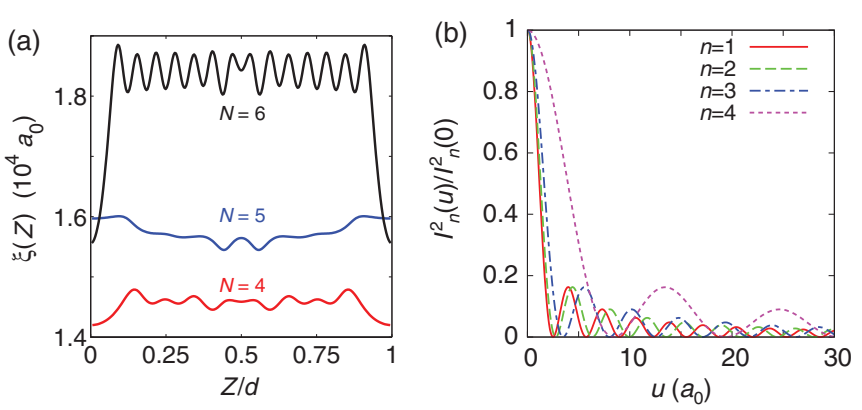

FIG. 5. (Color online) (a) Coherence lengths as a function of center of mass coordinate for nanofilms for thicknesses corresponding to four, five, and six (111) monolayers. The coherence lengths are shorter than in bulk $\mathrm{Al}\left(\sim 30236 a_{0}\right){ }^{28}$ (b) The mean-square radius of a subcondensate is determined mainly by how fast the functions $I_{n}^{2}(u)$ [cf. Eq. (43)] decay with $u$. The decay rates for the four subcondensates in a 3 (111) monolayer thick nanofilm are clearly different. The plot shows that the different functions $I_{n}(u)$ oscillate with different wavelengths.

interparticle distances indicated with vertical arrows in the figure. Thus, at certain distances, there is a tendency of pair depletion, while at other distances, the tendency is to a larger pair population. The amplitude modulation is a signature of interference and arises here because of the multicomponent character of the condensate. Indeed, it is readily shown that for sufficiently thin films (cf. Appendix B for details and analytical expressions), the Cooper pair density at the center of mass can be written $\varrho(Z)=\sum_{n} \varrho_{s}^{(n)}(Z)$, where $\varrho_{s}^{(n)}(Z)=\int d^{2} u d \zeta\left|\Psi_{n}(Z ; u, \zeta)\right|^{2}$ is the Cooper pair density of the $n$th subcondensate. This means that one has well-defined subcondensates, each consisting of pairs all belonging to a single band. One can see from Eqs. (42) and (43) that the $n$th subcondensate oscillates with a wavelength essentially determined by a narrow window of momenta around its Fermi momentum, $q_{\mathrm{F}}^{(n)}$ [see also the discussion on the function $I_{n}(u)$ at the end of this section and Fig. 5(b)]. Thus, in very thin nanofilms, where the Fermi momenta of the occupied bands are very different in magnitude, the interference pattern is strong. This interference pattern does not occur for a single component condensate.

It is of interest to see how the coherence length is affected by confinement and the multicomponent nature of the condensate. One can define the coherence length as the mean-square radius of the Cooper pair wave function ${ }^{22,47}$ around the pair center of mass. In inhomogeneous systems, it is a local rather than a global quantity. In our nanofilms, the relevant quantity is the mean square radius along the $x O y$ plane, namely,

$$
\xi^{2}(Z)=\frac{1}{\varrho_{s}(Z)} \int d^{2} u d \zeta u^{2}|\Psi(Z ; u, \zeta)|^{2},
$$

with $\varrho_{s}(Z)=\int d^{2} u d \zeta|\Psi(Z ; u, \zeta)|^{2}$ the Cooper pair density at the center of mass coordinates. To illustrate the spatial dependence of the coherence length and how it changes with nanofilm thickness, in Fig. 5(a), we show $\xi(Z)$ for nanofilms with thicknesses corresponding to $N=4,5$, and 6 monolayers grown in the [111] direction. On average, they are smaller than the bulk coherence length, which has a value of $\sim 30236 a_{0}{ }^{28}$ In the case of $N=4$, the coherence length magnitude is less than $50 \%$ of the bulk value. These smaller Cooper pair "sizes" reflect the fact that their average energy gap values are larger than their bulk counterpart $\left(2.8,2.7\right.$, and 2.1 times $\Delta^{b}$, for $N=4,5$, and 6, respectively).

The form of the oscillations in $\xi(Z)$ arises from the contributions of the different subcondensates, which have different gap values and different periodicity in $Z$. In this respect, it has recently been advanced that in two-gap superconductors, such as $\mathrm{MgB}_{2}$, the associated subcondensates present different coherence lengths near $T_{c} .{ }^{27}$ Very recently, a study based on the Bogoliubov-de Gennes equations has shown that the same should occur at $T=0 \mathrm{~K}$ in nanofilms a few monolayers thick. ${ }^{49}$ We also consider this question here. Within the present approach, a natural definition of the coherence length associated with the $n$th subcondensate is $\xi_{n}^{2}(Z)=\int d^{2} u d \zeta u^{2}\left|\Psi_{n}(Z ; u, \zeta)\right|^{2} / \varrho_{s}^{(n)}(Z)$. As shown in Appendix B, for $\Delta_{n} \ll \hbar \omega_{D}$, we have $\xi_{n} \simeq \hbar v_{\mathrm{F}}^{(n)} / 2 \sqrt{2} m \Delta_{n}$, where $v_{\mathrm{F}}^{(n)}$ is the Fermi velocity of the $n$th band. This is similar to the BCS expression for the coherence length in the three-dimensional case ${ }^{28}$ differing from it by a factor of order unity. Note that $\xi_{n}$ is a constant, independent of the center of mass coordinates.

We calculate, as an example, the coherence lengths in a two-monolayer thick nanofilm. It has two occupied bands, hence two subcondensates. At $T=0 \mathrm{~K}$, the two gap values are $\Delta_{1}=13.44 \Delta^{b} \mathrm{eV}$ and $\Delta_{2}=9.3 \Delta^{b} \mathrm{eV}$, and the corresponding coherence lengths are $\xi_{1} \simeq 4037 a_{0}$ and $\xi_{2} \simeq 4446 a_{0}$, respectively. Interestingly, we find that the ratio of the coherence lengths of two different subcondensates is generally rather insensitive to temperature. This is different from what is found for bulk two-gap superconductors within the (extended) Ginzburg-Landau formalism where the two coherence lengths tend to the same value in the $T \rightarrow T_{c}$ limit. ${ }^{27}$ Here, in the case of the two-monolayer nanofilm for $T \rightarrow T_{c}$, we have $\xi_{2} / \xi_{1}=1.15$. This reflects the fact that in sufficiently thin films, the subcondensates are well defined from the onset of the superconducting phase. Note also that the subcondensate with the larger gap has a shorter coherence length. The subcondensates in $\mathrm{MgB}_{2}$ and other two-gap superconductors show a similar behavior. ${ }^{27}$ However, this is not always the case in nanofilms, at least not in the present model. For example, consider the $N=4$ (111) monolayers nanofilm for which the coherence length is plotted in Fig. 5(a). In this case, there are five subcondensates, with gap values and coherence lengths given in Table I. One can see that $\Delta_{1}>\Delta_{2}>\Delta_{3}>\Delta_{4}>$ $\Delta_{5}$, but $\xi_{5}<\xi_{1}<\xi_{2}<\xi_{4}<\xi_{3}$. The reason for this is that $\xi_{n} \propto q_{\mathrm{F}}^{(n)} / \Delta_{n}$. Hence, although $\Delta_{n}>\Delta_{m}$ will favor $\xi_{n}<\xi_{m}$, the final outcome also depends on the relative magnitude of the corresponding Fermi momenta, $q_{\mathrm{F}}^{(n)}$ and $q_{\mathrm{F}}^{(m)}$, which are substantially different in ultrathin nanofilms. In fact, $\xi_{n}<\xi_{m}$ is equivalent to $\Delta_{m} / \Delta_{n}<q_{\mathrm{F}}^{(m)} / q_{\mathrm{F}}^{(n)}$.

TABLE I. Gap values and coherence lengths of the five subcondensates in a four (111) monolayers thick nanofilm.

\begin{tabular}{lccccc}
\hline \hline & $n=1$ & $n=2$ & $n=3$ & $n=4$ & $n=5$ \\
\hline$\Delta / \Delta^{b}$ & 3.65 & 3.40 & 2.95 & 2.47 & 1.58 \\
$\xi\left(a_{0}\right)$ & 14416 & 14653 & 15128 & 14698 & 13604 \\
\hline \hline
\end{tabular}


Finally, to get a feeling of the different decay lengths of the subcondensates, we consider the following. As we defined them, the coherence lengths reflect the "size" of the pair wave functions as a function of the interparticle distance, $u$, and they are essentially determined by the square of the function in Eq. (43), i.e., $I_{n}^{2}(u)$. As an example, in Fig. 5(b), we plot this quantity (normalized) for the subcondensates in the $N=3$ case of Fig. 4. The clearly different decay rates of the envelope functions for the different subcondensates already suggest different coherence lengths. In addition, the plot shows that the oscillation wavelength of the functions $I_{n}(u)$ is different for the different subcondensates. This illustrates the argument advanced above on this point.

\section{SUMMARY}

To summarize, we applied Anderson's approach to inhomogeneous superconductors, adapting Gor'kov's Green function formalism to inhomogeneous cases to study superconductivity in nanofilms. We find that in ultrathin nanofilms, it is very important to take into account the effects of confinement not only on electrons and phonons but also on the strength of the electron-phonon coupling. We find, indeed, that the effect of confinement on the screened electron-ion (background) interaction is crucial. Thus the quantum-well states first considered by Blatt and Thompson, who only took into account the effect of confinement on the electronic degrees of freedom, play only a partial role in determining the critical parameters. Furthermore, superconductivity in ultrathin nanofilms is typically multigap, with a condensate consisting of well defined subcondensates. The Cooper pair wave-function probability density as a function of interparticle distance along the nanofilm plane shows interference effects between the different subcondensates. Thus, at certain distances, the pair population is reduced, and at other distances, it tends to be enhanced. We also find that in such systems, the different subcondensates have different coherence lengths.

\section{ACKNOWLEDGMENTS}

This work was supported by the Flemish Science Foundation (FWO-Vl). R.S. thanks M. R. Norman, B. Sorée, and L. Komendová for useful comments.

\section{APPENDIX A: DIELECTRIC SCREENING IN A NANOFILM}

\section{Expansion of the bare Coulomb potential}

Given the potential

$$
v=v\left(\mathbf{r}, \mathbf{r}^{\prime}\right)=v\left(\mathbf{r}-\mathbf{r}^{\prime}\right)=\frac{1}{\left|\mathbf{r}-\mathbf{r}^{\prime}\right|},
$$

we consider the expansion over the single-electron basis of Sec. II,

$$
v\left(\mathbf{r}-\mathbf{r}^{\prime}\right)=\sum_{n \mathbf{q}} \sum_{n^{\prime} \mathbf{q}^{\prime}} \psi_{n \mathbf{q}}(\mathbf{r}) \psi_{n^{\prime} \mathbf{q}^{\prime}}^{*}\left(\mathbf{r}^{\prime}\right) v_{n \mathbf{q}, n^{\prime} \mathbf{q}^{\prime}} .
$$

The expansion coefficients are defined by

$$
v_{n \mathbf{q}, n^{\prime} \mathbf{q}^{\prime}}=\int d^{3} r d^{3} r^{\prime} v\left(\mathbf{r}-\mathbf{r}^{\prime}\right) \psi_{n \mathbf{q}}^{*}(\mathbf{r}) \psi_{n^{\prime} \mathbf{q}^{\prime}}\left(\mathbf{r}^{\prime}\right) .
$$

Because the system is translationally invariant in the $x O y$ plane, the first integral yields $v_{n \mathbf{q}, n^{\prime} \mathbf{q}^{\prime}}=v_{n n^{\prime} \mathbf{q}} \delta_{\mathbf{q}, \mathbf{q}^{\prime}}$. The second integral over the $x O y$ plane is solved using ${ }^{50}$

$$
\int d^{2} u \frac{e^{-i \mathbf{q} \cdot \mathbf{u}}}{\left(u^{2}+\left(z-z^{\prime}\right)^{2}\right)^{1 / 2}}=\frac{2 \pi}{q} e^{-q\left|z-z^{\prime}\right|} .
$$

The integrals over the $z$ axis are found to be

$$
\int_{0}^{d} d z d z^{\prime} e^{-q\left|z-z^{\prime}\right|} \sin \frac{n \pi z}{d} \frac{m \pi z^{\prime}}{d}=\frac{d}{q} f_{n m}(q),
$$

with

$$
\begin{aligned}
f_{n n^{\prime}}(q)= & \frac{\left[1+(-1)^{n+n^{\prime}}\right] \frac{n \pi}{q d} \frac{n^{\prime} \pi}{q d}}{\left[1+\left(\frac{n \pi}{q d}\right)^{2}\right]\left[1+\left(\frac{n^{\prime} \pi}{q d}\right)^{2}\right]} \frac{\left[1-(-1)^{n} e^{-q d}\right]}{q d} \\
& +\delta_{n n^{\prime}} \frac{1}{1+\left(\frac{n \pi}{q d}\right)^{2}}
\end{aligned}
$$

One finally has

$$
v_{n n^{\prime} \mathbf{q}}=f_{n n^{\prime}}(q) 4 \pi / q^{2} .
$$

Note that in the $d \rightarrow \infty$ limit, the coefficients tend well to the Fourier coefficients of the three-dimensional electron gas, i.e., $v_{n n^{\prime} \mathbf{q}} \rightarrow \delta_{n n^{\prime}} 4 \pi / q^{2}$. Also, the $1+(-1)^{n+n^{\prime}}$ factor in $f_{n n^{\prime}}(q)$ implies that coefficients with $n$ and $n^{\prime}$ of different parity will not contribute to the expansion in Eq. (A2). Furthermore, an analysis of the $q \rightarrow 0$ behavior shows that for $n, n^{\prime}$ both odd, $v_{n n^{\prime} \mathbf{q}} \propto 1 / q$, while for $n, n^{\prime}$ both even, $v_{n n^{\prime} \mathbf{q}}$ tends to a constant. Hence the long-range behavior of the potential is essentially represented by states that are symmetric with respect to the center of the nanofilm ( $n, n^{\prime}$ odd), with little contribution from states that are antisymmetric with respect to the center of the nanofilm.

\section{Polarization in the RPA}

Given the particle density operator in real space,

$$
\begin{aligned}
\hat{n}(\mathbf{r}) & =\sum_{\sigma} \hat{\psi}_{\sigma}^{\dagger}(\mathbf{r}) \hat{\psi}_{\sigma}(\mathbf{r}) \\
& =\sum_{\sigma} \sum_{\substack{n \mathbf{q} \\
n^{\prime} \mathbf{q}^{\prime}}} \psi_{n \mathbf{q}}^{*}(\mathbf{r}) \psi_{n^{\prime} \mathbf{q}^{\prime}}(\mathbf{r}) c_{n \mathbf{q} \sigma}^{\dagger} c_{n^{\prime} \mathbf{q}^{\prime} \sigma},
\end{aligned}
$$

its expansion coefficients in our single-electron basis, $\hat{n}_{n \mathbf{q}}=$ $\int d^{3} r \psi_{n \mathbf{q}}^{*}(\mathbf{r}) \hat{n}(\mathbf{r})$, can be readily shown to be

$$
\hat{n}_{n \mathbf{q}}=\frac{1}{\pi} \sqrt{\frac{2}{L^{2} d}} \sum_{m m^{\prime}} \sum_{\mathbf{p} \sigma} d_{n m m^{\prime}} c_{m \mathbf{p} \sigma}^{\dagger} c_{m^{\prime} \mathbf{p}+\mathbf{q} \sigma},
$$

with $d_{n m m^{\prime}}$ defined in Eq. (15). To solve for $\hat{n}_{n \mathbf{q}}$, one can exploit the linearity of the Heisenberg equation [cf. Eq. (25)]. Thus we consider first

$$
-i \hbar \frac{\partial}{\partial t} c_{m \mathbf{p} \sigma}^{\dagger} c_{m^{\prime} \mathbf{p}+\mathbf{q} \sigma}=\left[\hat{H}, c_{m \mathbf{p} \sigma}^{\dagger} c_{m^{\prime} \mathbf{p}+\mathbf{q} \sigma}\right] .
$$

The commutator with the noninteracting Hamiltonian is

$$
\begin{aligned}
{\left[\hat{H}_{0}, c_{m \mathbf{p} \sigma}^{\dagger} c_{m^{\prime} \mathbf{p}+\mathbf{q} \sigma}\right] } & =\left(E_{m \mathbf{p}}-E_{m^{\prime} \mathbf{p}+\mathbf{q}}\right) c_{m \mathbf{p} \sigma}^{\dagger} c_{m^{\prime} \mathbf{p}+\mathbf{q} \sigma} \\
& =\left(\epsilon_{m \mathbf{p}}-\epsilon_{m^{\prime} \mathbf{p}+\mathbf{q}}\right) c_{m \mathbf{p} \sigma}^{\dagger} c_{m^{\prime} \mathbf{p}+\mathbf{q} \sigma},
\end{aligned}
$$

where, for practical reasons, we introduce the energies referenced to the Fermi level (e.g., $\epsilon_{m \mathbf{p}}=E_{m \mathbf{p}}-\mu$ ). The 
commutator with the potential energy operator is calculated in the random phase approximation (RPA),

$$
\begin{aligned}
{\left[\hat{V}, c_{m \mathbf{p} \sigma}^{\dagger} c_{m^{\prime} \mathbf{p}+\mathbf{q} \sigma}\right]=} & -e \frac{1}{\pi} \sqrt{\frac{2}{L^{2} d}} \sum_{l \mathbf{k}} V_{l \mathbf{k}}\left(\sum_{j} d_{l j m} c_{j \mathbf{k}+\mathbf{p} \sigma}^{\dagger}\right. \\
& \left.\times c_{m^{\prime} \mathbf{p}+\mathbf{q} \sigma}-\sum_{j^{\prime}} d_{l m^{\prime} j^{\prime}} c_{m \mathbf{p} \sigma}^{\dagger} c_{j^{\prime} \mathbf{p}+\mathbf{q}-\mathbf{k} \sigma}\right) \\
\stackrel{\mathrm{RPA}}{\simeq} & -e \frac{1}{\pi} \sqrt{\frac{2}{L^{2} d}} \sum_{l} V_{l \mathbf{q}} d_{l m m^{\prime}}\left(c_{m^{\prime} \mathbf{p}+\mathbf{q} \sigma}^{\dagger}\right. \\
& \left.\times c_{m^{\prime} \mathbf{p}+\mathbf{q} \sigma}-c_{m \mathbf{p} \sigma}^{\dagger} c_{m \mathbf{p} \sigma}\right) .
\end{aligned}
$$

Inserting the results for the commutators in Eq. (A10), and taking the Fourier transform in time, results in

$$
\begin{aligned}
&(-\hbar \omega\left.-\epsilon_{m \mathbf{p}}+\epsilon_{m^{\prime} \mathbf{p}+\mathbf{q}}\right) c_{m \mathbf{p} \sigma}^{\dagger} c_{m^{\prime} \mathbf{p}+\mathbf{q} \sigma} \\
&=-e \frac{1}{\pi} \sqrt{\frac{2}{L^{2} d}} \sum_{l} V_{l \mathbf{q}}(\omega) d_{l m m^{\prime}} \\
& \quad \times\left(c_{m^{\prime} \mathbf{p}+\mathbf{q} \sigma}^{\dagger} c_{m^{\prime} \mathbf{p}+\mathbf{q} \sigma}-c_{m \mathbf{p} \sigma}^{\dagger} c_{m \mathbf{p} \sigma}\right)
\end{aligned}
$$

from which

$$
\begin{aligned}
c_{m \mathbf{p} \sigma}^{\dagger} c_{m^{\prime} \mathbf{p}+\mathbf{q} \sigma}= & e \frac{1}{\pi} \sqrt{\frac{2}{L^{2} d}} \sum_{l} V_{l \mathbf{q}}(\omega) d_{l m m^{\prime}} \\
& \times \frac{c_{m^{\prime} \mathbf{p}+\mathbf{q} \sigma}^{\dagger} c_{m^{\prime} \mathbf{p}+\mathbf{q} \sigma}-c_{m \mathbf{p} \sigma}^{\dagger} c_{m \mathbf{p} \sigma}}{\hbar \omega-\epsilon_{m^{\prime} \mathbf{p}+\mathbf{q}}+\epsilon_{m \mathbf{p}}} .
\end{aligned}
$$

With the latter result one can determine at once the density in Eq. (A9). Furthermore, given that the induced charge density is given by the charge times the particle (ground-state) average, $\varrho_{n \mathbf{q}}(\omega)=-e\left\langle\hat{n}_{n \mathbf{q}}(\omega)\right\rangle$, one has

$$
\begin{aligned}
\varrho_{n \mathbf{q}}(\omega)= & \frac{2 e^{2}}{\pi^{2} L^{2} d} \sum_{m m^{\prime}} d_{n m m^{\prime}} d_{l m m^{\prime}} V_{l \mathbf{q}}(\omega) \\
& \times \sum_{\mathbf{p} \sigma} \frac{n_{\mathrm{F}}\left(\epsilon_{m \mathbf{p} \sigma}\right)-n_{\mathrm{F}}\left(\epsilon_{m^{\prime} \mathbf{p}+\mathbf{q} \sigma}\right)}{\hbar \omega-\epsilon_{m^{\prime}} \mathbf{p}+\mathbf{q}}+\epsilon_{m \mathbf{p}}
\end{aligned}
$$

with $n_{\mathrm{F}}$ the Fermi occupation function already introduced in Sec. III B. Defining the RPA polarization by

$$
\begin{aligned}
P_{n l \mathbf{q}}^{(1)}(\omega)= & \frac{2}{\pi^{2} L^{2} d} \sum_{m m^{\prime}} d_{n m m^{\prime}} d_{l m m^{\prime}} \\
& \times \sum_{\mathbf{p} \sigma} \frac{n_{\mathrm{F}}\left(\epsilon_{m \mathbf{p} \sigma}\right)-n_{\mathrm{F}}\left(\epsilon_{m^{\prime} \mathbf{p}+\mathbf{q} \sigma}\right)}{\hbar \omega-\epsilon_{m^{\prime} \mathbf{p}+\mathbf{q}}+\epsilon_{m \mathbf{p}}},
\end{aligned}
$$

the charge density can immediately be written as the matrix product in Eq. (27), i.e., $\varrho_{n \mathbf{q}}(\omega)=e^{2} \sum_{l} P_{n l \mathbf{q}}^{(1)}(\omega) V_{l \mathbf{q}}(\omega)$.

\section{Static screening in the long-wavelength limit}

In the present work, we are interested in the static screening in the long-wavelength limit. Thus $P_{n m \mathbf{q}}^{(1)}(\omega)$ for $\omega=0$ and $q \rightarrow 0$ is calculated in the following. First, a little algebra shows that one can write (the eigenenergies do not depend on spin, so the $\sigma$ subindex can be dropped)

$$
\begin{aligned}
\frac{n_{\mathrm{F}}\left(\epsilon_{n \mathbf{p}}\right)-n_{\mathrm{F}}\left(\epsilon_{m \mathbf{p}+\mathbf{q}}\right)}{\hbar \omega-\epsilon_{m \mathbf{p}+\mathbf{q}}+\epsilon_{n \mathbf{p}}} & \frac{n_{\mathrm{F}}\left(\epsilon_{n \mathbf{p}}\right)\left[1-n_{\mathrm{F}}\left(\epsilon_{m \mathbf{p}+\mathbf{q}}\right)\right]+n_{\mathrm{F}}\left(\epsilon_{m \mathbf{p}+\mathbf{q}}\right)\left[n_{\mathrm{F}}\left(\epsilon_{n \mathbf{p}}\right)-1\right]}{\hbar \omega-\epsilon_{m \mathbf{p}+\mathbf{q}}+\epsilon_{n \mathbf{p}}} \\
= & n_{\mathrm{F}}\left(\epsilon_{n \mathbf{p}}\right)\left[1-n_{\mathrm{F}}\left(\epsilon_{m \mathbf{p}+\mathbf{q}}\right)\right] \\
\quad & \times\left(\frac{1}{\hbar \omega-\epsilon_{m \mathbf{p}+\mathbf{q}}+\epsilon_{n \mathbf{p}}}-\frac{1}{\hbar \omega+\epsilon_{m \mathbf{p}+\mathbf{q}}-\epsilon_{n \mathbf{p}}}\right) .
\end{aligned}
$$

(For the above, recall that $\epsilon_{n-\mathbf{p}}=\epsilon_{n \mathbf{p}}$.) So the polarization is rewritten as

$$
\begin{aligned}
P_{n m \mathbf{q}}^{(1)}(\omega)= & \frac{A^{2}}{\pi^{2}} \sum_{l l^{\prime}} d_{n l l^{\prime}} d_{m l l^{\prime}} \sum_{\mathbf{p} \sigma} n_{\mathrm{F}}\left(\epsilon_{l \mathbf{p}}\right)\left[1-n_{\mathrm{F}}\left(\epsilon_{l^{\prime} \mathbf{p}+\mathbf{q}}\right)\right] \\
& \times \frac{2\left(\epsilon_{l^{\prime} \mathbf{p}+\mathbf{q}}-\epsilon_{l \mathbf{p}}\right)}{(\hbar \omega)^{2}-\left(\epsilon_{l^{\prime} \mathbf{p}+\mathbf{q}}+\epsilon_{l \mathbf{p}}\right)^{2}} .
\end{aligned}
$$

For $\omega=0$, the summation over $\mathbf{p}$ (in the $L \rightarrow \infty$ limit) requires the following result:

$$
\begin{gathered}
\int_{\substack{|\mathbf{p}|<k_{\mathrm{F}}^{(l)} \\
|\mathbf{p}+\mathbf{q}|>k_{\mathrm{F}}}} d^{2} p \frac{1}{\left(l^{\prime}\right)} \frac{1}{\left.l^{2}\right) \frac{\pi^{2}}{d^{2}}+2 \mathbf{p} \cdot \mathbf{q}+q^{2}} \\
\stackrel{l^{q} \rightarrow 0}{\equiv} s_{l l^{\prime}}= \begin{cases}0 & l^{\prime}=l, \\
\frac{\pi}{2} & l_{\max }>l^{\prime}>l, \\
\pi & l^{\prime}>l_{\max }, \\
\pi \frac{\left.k_{\mathrm{F}}^{(l)}\right)^{2}}{\left(l^{\prime 2}-l^{2}\right) \frac{\pi^{2}}{d^{2}}} & \end{cases}
\end{gathered}
$$

where $l_{\max }$ is the index of the highest occupied band. ${ }^{51}$ Hence one finds that in the limit of small momentum, the polarization is a constant given by $P_{m l \mathbf{q} \rightarrow 0}^{(1)}(0)=$ $\left(4 m / \pi^{4} \hbar^{2} d\right) \sum_{l l^{\prime}} d_{n l l^{\prime}} d_{m l l^{\prime}} s_{l l^{\prime}}$. Thus, in the above limit, the momentum dependence of the static dielectric function for small $\mathbf{q}$ is given essentially by the Coulomb potential, i.e.,

$$
\varepsilon_{n m \mathbf{q}}(0) \simeq \delta_{n m}-e^{2} \sum_{m} v_{n m \mathbf{q}} P_{m l \mathbf{q} \rightarrow 0}^{(1)}(0) .
$$

For this very reason, the long-wavelength limit of the static, screened Coulomb potential, $v_{m n^{\prime} \mathbf{q} \rightarrow 0}^{\mathrm{scr}}(0)=$ $\lim _{q \rightarrow 0} \sum_{n} \varepsilon_{m n \mathbf{q}}^{-1}(0) v_{n n^{\prime} \mathbf{q}}$, is nondivergent.

\section{APPENDIX B: COOPER PAIR DENSITY AND COHERENCE LENGTH IN NANOFILMS}

The Cooper pair density and coherence length as a function of the center of mass coordinates have a simple form in ultrathin nanofilms. To calculate the Cooper pair density,

$$
\varrho_{s}(Z)=\int d^{2} u d \zeta|\Psi(Z ; u, \zeta)|^{2}
$$


requires one to perform the integral

$$
\int_{0}^{\infty} d u и I_{n}(u) I_{m}(u)
$$

with $I_{n}(u)$ given by Eq. (43). This integral is equivalent to

$$
\int_{k_{\min }^{(n)}}^{k_{\max }^{(n)}} d k \int_{q_{\min }^{(n)}}^{q_{\max }^{(n)}} d q k \frac{1}{\sqrt{\left(\epsilon_{k n}^{2}+\Delta_{n}^{2}\right)\left(\epsilon_{q m}^{2}+\Delta_{m}^{2}\right)}} \delta(k-q) .
$$

This expression is obtained making use of $x J_{0}(x)=\left[x J_{1}(x)\right]^{\prime}$, and the Dirac $\delta$ function arises because of the completeness relation of the Bessel functions. ${ }^{52}$ Now, for sufficiently thin nanofilms [e.g., for $d \leqslant 75 a_{0}$, or 17 (111) monolayers, for the electron density considered in this work] and any two bands, one has $\left|\epsilon_{k n}-\epsilon_{k m}\right|>2 \hbar \omega_{D}$. This means that the intervals $\left[k_{\min }^{(n)}, k_{\max }^{(n)}\right]$ and $\left[q_{\min }^{(m)}, q_{\max }^{(m)}\right]$ do not overlap. Then, it is easily shown that the Cooper pair density reduces to the form $\varrho_{s}(Z)=$ $\sum_{n} \varrho_{s}^{(n)}(Z)$, i.e., as mentioned in Sec. V, the condensate is comprised of well-defined subcondensates, with all pairs in any one of them belonging to the same band. The Cooper pair density of the $n$th subcondensate is given by (to simplify the expression below, we assume that the Fermi level, $\mu$, does not fall within the energy window $\left.\left[-\hbar \omega_{D},+\hbar \omega_{D}\right]\right)$

$$
\varrho_{s}^{(n)}(Z)=\frac{m / \hbar^{2}}{8 \pi^{3} d} \Delta_{n} C_{n}(Z) \tan ^{-1}\left(\frac{\hbar \omega_{D}}{\Delta_{n}}\right) .
$$

The $\tan ^{-1}$ term arises from the above integral, while the function $C_{n}(Z)$ comes from the integral over $\zeta$ in the definition of $\varrho_{s}$ and is given by

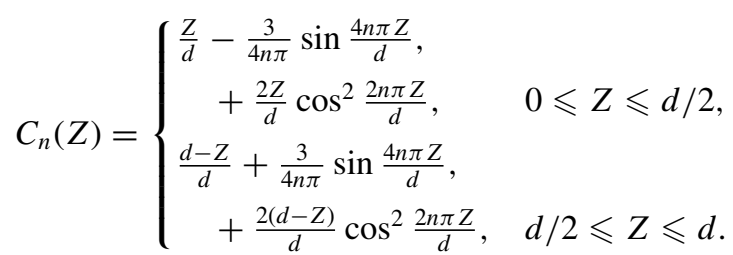

The coherence length at the center of mass coordinates, defined in Eq. (44), is found to be written as a summation over occupied bands as

$$
\xi^{2}(Z)=\frac{\left(16 \pi^{3} d\right)^{-1}}{\varrho_{s}(Z)} \sum_{n} K_{n} C_{n}(Z) / \Delta_{n},
$$

with

$$
K_{n}=\frac{\left(\hbar q_{\mathrm{F}}^{(n)}\right)^{2}}{4 m}\left[\tan ^{-1}\left(\frac{\hbar \omega_{D}}{\Delta_{n}}\right)+\frac{\left(\frac{\hbar \omega_{D}}{\Delta_{n}}\right)^{3}-\frac{\hbar \omega_{D}}{\Delta_{n}}}{\left(1+\frac{\hbar \omega_{D}^{2}}{\Delta_{n}^{2}}\right)^{2}}\right] .
$$

From the above, one can see that the dependence of the coherence length on $Z$ is due to the existence of several subcondensates. Indeed, if there were only one condensate (i.e., only one band occupied), Eqs. (B4) and (B5) show that the coherence length would be constant. In fact, as discussed before, one can also consider the coherence length of the $n$th subcondensate, which we define by $\xi_{n}^{2}(Z)=$ $\int d^{2} u d \zeta u^{2}\left|\Psi_{n}(Z ; u, \zeta)\right|^{2} / \varrho_{s}^{(n)}(Z)$. This simply gives

$$
\xi_{n}^{2}=\frac{\hbar^{2}}{2 m} \frac{K_{n}}{\Delta_{n}^{2} \tan ^{-1}\left(\hbar \omega_{D} / \Delta_{n}\right)} .
$$

Thus the coherence length of the subcondensates is constant, as one might have expected. The total coherence length can then be written as a weighted sum of the subcondensate coherence lengths, $\xi^{2}(Z)=\sum_{n} \varrho_{s}^{(n)}(Z) \xi_{n}^{2} / \varrho_{s}(Z)$. Also, given that one typically has $\Delta_{n} \ll \hbar \omega_{D}$, it is straightforward to show that to lowest order in $\Delta_{n} / \hbar \omega_{D}$, one has

$$
\xi_{n}^{2} \simeq \frac{1}{2}\left(\frac{\hbar^{2} q_{\mathrm{F}}^{(n)}}{2 m \Delta_{n}}\right)^{2}\left(1+\frac{2}{\pi} \frac{\Delta_{n}}{\hbar \omega_{D}}\right) .
$$

This immediately leads to the expression given in Sec. V, $\xi_{n}=\hbar v_{\mathrm{F}}^{(n)} / m \Delta_{n} 2 \sqrt{2}$ (where $v_{\mathrm{F}}^{(n)}$ denotes the Fermi velocity of band $n$ ).

Incidentally, from the Cooper pair density, the total superfluid density in the nanofilm, defined by

$$
n_{s}=\frac{1}{V} \int d^{2} R d Z \varrho(Z),
$$

can be obtained and is found to be given simply by

$$
n_{s}=\frac{m / \hbar^{2}}{16 \pi^{3} d} \sum_{n} \Delta_{n} \tan ^{-1} \frac{\hbar \omega_{D}}{\Delta_{n}} .
$$

As in the nanowire case (cf. I), we find that $n_{s}$ is several orders of magnitude smaller than the total electron density. This appears to be a characteristic of confined weak coupling superconductors, bringing another distinction with respect to bulk weak coupling superconductors, where virtually all the normal state electrons are believed to contribute to the superfluid density. ${ }^{53}$
${ }^{1}$ J. M. Blatt and C. J. Thompson, Phys. Rev. Lett. 10, 332 (1963).

${ }^{2}$ C. J. Thompson and J. M. Blatt, Phys. Lett. 5, 6 (1963).

${ }^{3}$ P. W. Anderson, J. Phys. Chem. Solids 11, 26 (1959).

${ }^{4}$ K. Tanaka and F. Marsiglio, Phys. Rev. B 62, 5345 (2000).

${ }^{5}$ M. Yu, M. Strongin, and A. Paskin, Phys. Rev. B 14, 996 (1976).

${ }^{6}$ B. Chen, Z. Zhu, and X. C. Xie, Phys. Rev. B 74, 132504 (2006).

${ }^{7}$ E. H. Hwang, S. Das Sarma, and M. A. Stroscio, Phys. Rev. B 61, 8659 (2000).
${ }^{8}$ A. F. Hebard and A. T. Fiory, Phys. Rev. Lett. 44, 291 (1980).

${ }^{9}$ B. G. Orr, H. M. Jaeger, and A. M. Goldman, Phys. Rev. Lett. 53, 2046 (1984).

${ }^{10}$ Y. Guo, Y.-F. Zhang, X.-Y. Bao, T.-Z. Han, Zhe Tang, L.-X. Zhang, W.-G. Zhu, E. G. Wang, Q. Niu, Z. Q. Qiu, J.-F. Jia, Z.-X. Zhao, and Q.-K. Xue, Science 306, 1915 (2004).

${ }^{11}$ D. Eom, S. Qin, M.-Y. Chou, and C. K. Shih, Phys. Rev. Lett. 96, 027005 (2006).

${ }^{12}$ S. Qin, J. Kim, Q. Niu, and C.-K. Shih, Science 324, 1314 (2009). 
${ }^{13}$ T. Zhang, P. Cheng, W.-J. Li, Y.-J. Sun, G. Wang, X.-G. Zhu, K. He, L. Wang, X. Ma, X. Chen, Y. Wang, Y. Liu, H.-Q. Lin, J.-F. Jia, and Q.-K. Xue, Nat. Phys. 6, 104 (2010).

${ }^{14}$ N. A. Court, A. J. Ferguson, and R. G. Clark, Supercond. Sci. Technol. 21, 015013 (2008).

${ }^{15}$ A. A. Shanenko, M. D. Croitoru, and F. M. Peeters, Europhys. Lett. 76, 498 (2006).

${ }^{16}$ F. M. Peeters, A. A. Shanenko, and M. D. Croitoru, in Handbook of Nanophysics, edited by Klaus D. Sattler (Taylor and Francis, CRC Press, 2010).

${ }^{17}$ Y. Chen, A. A. Shanenko, and F. M. Peeters, Phys. Rev. B 85, 224517 (2012).

${ }^{18}$ G. Q. Huang, Phys. Rev. B 78, 214514 (2008).

${ }^{19}$ J. Noffsinger and M. L. Cohen, Phys. Rev. B 81, 214519 (2010).

${ }^{20}$ L. P. Gor'kov, Sov. Phys. JETP 7, 505 (1958).

${ }^{21}$ R. Saniz, B. Partoens, and F. M. Peeters, Phys. Rev. B 85, 144504 (2012).

${ }^{22}$ N. Pillet, N. Sandulescu, and P. Schuck, Phys. Rev. C 76, 024310 (2007).

${ }^{23}$ A. Pastore, F. Barranco, R. A. Broglia, and E. Vigezzi, Phys. Rev. C 78, 024315 (2008).

${ }^{24}$ S. H. Pan, J. P. O'Neal, R. L. Badzey, C. Chamon, H. Ding, J. R. Engelbrecht, Z. Wang, H. Eisaki, S. Uchida, A. K. Guptak, K.-W. Ngk, E. W. Hudson, K. M. Lang, and J. C. Davis, Nature (London) 413, 282 (2001)

${ }^{25}$ K. K. Gomes, A. N. Pasupathy, A. Pushp, S. Ono, Y. Ando, and A. Yazdani, Nature (London) 447, 569 (2007).

${ }^{26}$ A. E. Koshelev and A. A. Golubov, Phys. Rev. Lett. 90, 177002 (2003).

${ }^{27}$ L. Komendová, M. V. Milošević, A. A. Shanenko, and F. M. Peeters, Phys. Rev. B 84, 064522 (2011).

${ }^{28}$ A. L. Fetter and J. D. Walecka, Quantum Theory of Many-Particle systems, (McGraw-Hill, New York, 1971).

${ }^{29}$ G. D. Mahan, Many-Particle Physics (Plenum Press, New York, 1981).

${ }^{30}$ The interaction is supplemented with the subsidiary condition that the interaction is restricted to states within an energy window of width $2 \hbar \omega_{D}$ around the Fermi level, where $\omega_{D}$ is the Debye frequency. Then, for systems in which $T_{c} \ll \hbar \omega_{D} / k_{B}$, one essentially has $T_{c} \propto e^{-1 / \mathcal{N}(0) g}$, where $\mathcal{N}(0)$ is the electron density of states per spin at the Fermi level, see Ref. 28.

${ }^{31}$ The displacement verifies the "clamped surface" boundary conditions, i.e., unrestricted normal component of the stress tensor and zero displacement, see, e.g., Ref. 32.

${ }^{32}$ M. A. Stroscio and M. Dutta, Phonons in Nanostructures (Cambridge University Press, Cambridge, 2001).

${ }^{33}$ W. H. Backes, F. M. Peeters, F. Brosens, and J. T. Devreese, Phys. Rev. B 45, 8437 (1992).

${ }^{34}$ Recall that $\delta\left(z-z^{\prime}\right)=\frac{2}{\pi} \sum_{n} \sin (n z) \sin \left(n z^{\prime}\right)$.

${ }^{35}$ Note that $\gamma_{m}$ with $m$ odd (even) corresponds to phonon modes symmetric (antisymmetric) with respect to the center of film.
A closer inspection of the $d_{m n n^{\prime}}$ reveals that symmetric phonon modes can only couple electron states of the same parity, while antisymmetric phonon modes couple states with different parity only. A similar result was obtained in Ref. 7. In their formulation, however, electron-phonon coupling is represented by a single (bulk) value.

${ }^{36}$ In their first work, ${ }^{1}$ Blatt and Thompson use a contact potential as well, but the fact that their single-electron states are obtained in a self-consistent fashion results in effective interaction matrix elements that slightly lift the degeneracy between gap values. In Ref. 2, they drop the self-consistency scheme and find a single gap value.

${ }^{37}$ N. W. Ashcroft and N. D. Mermin, Solid State Physics (Saunders College, Philadelphia, 1976).

${ }^{38}$ A. Balandin and K. L. Wang, Phys. Rev. B 58, 1544 (1998).

${ }^{39}$ S. Schmidt and C. A. Grimes, Sens. Actuators A 94, 189 (2001).

${ }^{40}$ B. Feng, Z. Li, and X. Zhang, J. Appl. Phys. 105, 104315 (2009).

${ }^{41}$ The results in Ref. 40 indicate that the change in sound velocity in films with thicknesses within a few-nanometer range is of the order of $10 \%$. Such an effect would not change the values of our calculated critical parameters significantly and would not affect the behavior of these parameters as a function of film thickness, which is what we focus on.

${ }^{42}$ An explanation of the results in Ref. 14 can be found in Ref. 16

${ }^{43}$ MATLAB 7.6.0.324 (R2008a), The MathWorks Inc., Natick, MA, 2008.

${ }^{44}$ For $n$ even, $v_{n n q \rightarrow 0}^{\text {scr }}$ changes more weakly with the number of occupied bands for the following reason. As discussed in Sec. III A, for $\mathbf{q} \rightarrow 0$ the matrix elements of the dielectric function with even indices depend comparatively little on the polarization, and hence on the number of occupied bands. Thus the matrix elements of $v^{\text {scr }}=\varepsilon^{-1} v$ with even indices also have this property.

${ }^{45}$ G. D. Mahan, Phys. Rev. B 48, 16557 (1993).

${ }^{46}$ As $d \rightarrow \infty$, the quantum number $n \pi / d$ becomes a momentum in the $z$ direction, and the set of disjoint $n$-dependent circumferences defining the Fermi surface merge into the single Fermi surface sphere.

${ }^{47}$ A. J. Leggett, in Superconductivity, edited by K. H. Bennemann and J. B. Ketterson (Springer-Verlag, Berlin, 2008), Vol. II.

${ }^{48}$ A. J. Leggett, Rev. Mod. Phys. 47, 331 (1975).

${ }^{49}$ Y. Chen, A. A. Shanenko, A. Perali, and F. M. Peeters, J. Phys: Condens. Matter 24, 185701 (2012).

${ }^{50}$ P. B. Visscher and L. M. Falicov, Phys. Rev. B 3, 2541 (1971).

${ }^{51}$ Use is made of the result $\int_{0}^{2 \pi} d \theta(b \cos \theta)^{-1}=2 \pi / \sqrt{a^{2}-b^{2}}$, with $a>b>0$, which can be shown by contour integration.

${ }^{52}$ Handbook of Mathematical Functions, edited by M. Abramowitz and I. Stegun (Dover, New York, 1972).

${ }^{53}$ M. Tinkham, Introduction to Superconductivity (McGraw-Hill, New York, 1996). 